Mean- $f$ i el $d$ i nt er act i ng boson $r$ andom poi $n t$ fi el ds in weak harmoni $c$ traps

\begin{tabular}{|l|l|}
\hline 著者 & Tamur a H r oshi, Zagr ebnov Val ent i n A. \\
\hline $\begin{array}{l}\text { j ournal or } \\
\text { publ i cat i on t i t l e }\end{array}$ & Journal of Nat hemat i cal Physi cs \\
\hline vol une & 50 \\
\hline page $r$ ange & 23301 \\
\hline year & $2009-02-09$ \\
\hline URL & ht t p: //hdl . handl e. net /2297/14523 \\
\hline
\end{tabular}




\title{
Mean-field interacting boson random point fields in weak harmonic traps
}

\author{
Hiroshi Tamura ${ }^{1, a)}$ and Valentin A. Zagrebnov ${ }^{2, b)}$ \\ ${ }^{1}$ Graduate School of the Natural Science and Technology, Kanazawa University, \\ Kanazawa 920-1192, Japan \\ ${ }^{2}$ Université de la Méditerranée (Aix-Marseille II) and Centre de Physique Théorique, \\ UMR 6207 CNRS, Luminy, Case 907, 13288 Marseille Cedex 9, France
}

(Received 16 July 2008; accepted 29 December 2008; published online 2 February 2009)

\begin{abstract}
A model of the mean-field interacting boson gas trapped by a weak harmonic potential is considered by the boson random point field methods. We prove that in the weak harmonic trap limit there are two phases distinguished by the boson condensation and by a different behavior of the local particle density. For chemical potentials less than a certain critical value, the resulting random point field (RPF) coincides with the usual boson RPF, which corresponds to a noninteracting (ideal) boson gas. For the chemical potentials greater than the critical value, the boson RPF describes a divergent (local) density, which is due to localization of the macroscopic number of condensed particles. Notice that it is this kind of transition that observed in experiments producing the Bose-Einstein condensation in traps. (C) 2009 American Institute of Physics. [DOI: 10.1063/1.3072911]
\end{abstract}

\section{INTRODUCTION AND MAIN RESULTS}

\section{A. Weak harmonic traps}

We consider the quantum statistical mechanical models of boson gas equipped with a $\kappa$-parametrized family of one-particle Hamiltonians of harmonic oscillators

$$
h_{\kappa}=\frac{1}{2} \sum_{j=1}^{d}\left(-\frac{\partial^{2}}{\partial x_{j}^{2}}+\frac{x_{j}^{2}}{\kappa^{2}}-\frac{1}{\kappa}\right),
$$

which are self-adjoint operators in the Hilbert space $\mathfrak{H}:=L^{2}\left(\mathbb{R}^{d}\right)$.

In this setup a "thermodynamic limit" corresponds to $\kappa \rightarrow \infty$ (i.e., the "opening" of the $\operatorname{trap}^{3}$ ), which we call the weak harmonic trap (WHT) limit. Notice that the set $C_{0}^{\infty}\left(\mathrm{R}^{d}\right)$ is a form core of operator (1.1) and that this set is also a form core for the operator $(-\Delta) / 2$. Here $\Delta$ is the standard Laplace operator in $\mathbb{R}^{d}$. Then (see, e.g., Ref. 7) one obtains the strong resolvent convergence,

$$
\lim _{\kappa \rightarrow \infty} h_{\kappa}=(-\Delta) / 2 \text {. }
$$

In spite of convergence (1.2), there is a drastic difference between the properties of the infinite ideal boson gas (IBG) systems prepared via the WHT limit and via standard thermodynamic limit (TDL) $\lim _{L \rightarrow \infty} \Lambda_{L}=\mathbb{R}^{d}$ with the one-particle operators $t_{L}:=(-\Delta / 2)_{L}$ with a "nonsticky" (e.g., Dirichlet) boundary conditions. ${ }^{21}$ Here $\Lambda_{L=1} \subset \mathbb{R}^{d}$ is a bounded open region of unit volume $\left|\Lambda_{L=1}\right|$ $=1$ containing the origin $x=0$ whose boundary $\partial \Lambda_{L=1}$ is piecewise continuously differentiable and

\footnotetext{
${ }^{a)}$ Electronic mail: tamurah@kenroku.kanazawa-u.ac.jp.

${ }^{b)}$ Electronic mail: zagrebnov@cpt.univ-mrs.fr. 


$$
\Lambda_{L}:=\left\{x \in \mathbb{R}^{d} \mid L^{-1} x \in \Lambda_{L=1}\right\}, \quad L>0 .
$$

In fact, it is known that the Bose-Einstein condensation (BEC) occurs for dimensions $d>1$ in the IBG via WHT limit $\kappa \rightarrow \infty$, while for dimensions $d>2$ in the IBG via TDL, see (1.13) and Refs. 3 and 14. Similarly, it is well known that thermodynamic properties of the boson gases are very sensible to different ways of taking the thermodynamic limit ${ }^{9}$ or to the different choices of the boundary conditions. $^{21}$

The purpose of this paper is to examine the position distribution of the mean-field boson model in WHT limit and to compare its behavior to those of the mean-field boson models in TDL or ideal boson models in WHT limit.

Our method is based on the theory of random point fields (RPFs) (see, e.g., Ref. 1). The usual boson and the fermion RPFs ${ }^{11-13}$ have been formulated in a unified way in terms of the Fredholm determinant together with other related RPFs, which are indexed by fractional numbers, in Ref. 17. They have been rederived as theories which describe position distributions of the constituent particles of quantum gases in the thermodynamic limit for canonical ensembles in Ref. 18. It was shown that the RPF, corresponding to fractional numbers, ${ }^{17}$ describes the gases which consist of particles obeying the parastatistics. ${ }^{20}$ The RPF describing a homogeneous Bose-Einstein condensation has been studied for the first time in Ref. 19, where the RPF is given by the convolution of usual boson RPF and another RPF. The latter one seems to describe position distribution of the condensed part of the constituent bosons. This RPF has been reformulated using the Cox process. ${ }^{4}$

These theories of RPFs yield a precise information about the position distribution of the constituent quantum particles, although they are not suitable to characterize the quantum systems completely (however, see, Refs. 5 and 6).

\section{B. Ideal and mean-field boson gases in the WHT}

Recall that in the grand-canonical Gibbs ensemble the partition function of the IBG trapped by harmonic potential (1.1) is given by

$$
\Xi_{0, \kappa}(\beta, \mu):=\sum_{n=0}^{\infty} e^{\beta \mu n} \operatorname{Tr} \mathfrak{H}_{\mathrm{symm}}^{n}\left[\otimes^{n} G_{\kappa}(\beta)\right] .
$$

Here $\mathfrak{H}_{\text {symm }}^{n}:=\left(\otimes^{n} L^{2}\left(\mathbb{R}^{d}\right)\right)_{\text {symm }}$ is the $n$-fold symmetric Hilbert space tensor product of $\mathfrak{H}$ $:=L^{2}\left(\mathbb{R}^{d}\right), G_{\kappa}(\beta)=e^{-\beta h_{\kappa}}$ the one-particle Gibbs semigroup. ${ }^{23}$ The zeroth term in (1.4) equal to 1 by definition. We consider the case of positive inverse temperature $\beta>0$ and of negative chemical potential $\mu<0$.

The spectrum of operator (1.1) is discrete and has the form

$$
\operatorname{spec}\left(h_{\kappa}\right)=\left\{\epsilon_{\kappa}(s):=|s|_{1} / \kappa \mid s=\left(s_{1}, \ldots, s_{d}\right) \in \mathbb{Z}_{+}^{d}\right\},
$$

where $|s|_{1}:=\sum_{j=1}^{d} s_{j}$ and $\mathbb{Z}_{+}^{1}$ is the set of all non-negative integers. The normalized eigenfunctions of the states for $s \in \mathbb{Z}_{+}^{d}$ are given by

$$
\phi_{s, \kappa}(x)=\frac{1}{\kappa^{d / 4}} \phi_{s}(x / \sqrt{\kappa})=\prod_{j=1}^{d} \frac{1}{\kappa^{1 / 4}} \phi_{s_{j}}\left(x_{j} / \sqrt{\kappa}\right),
$$

where for each component $j, \phi_{s_{j}}$ is related to the Hermite polynomials $H_{s_{j}}(z)$ by

$$
\phi_{s_{j}}\left(x_{j}\right)=\left(2^{s_{j}} s_{j} ! \sqrt{\pi}\right)^{-1 / 2} H_{s_{j}}\left(x_{j}\right) e^{-x_{j}^{2} / 2}, \quad j=1, \ldots, d .
$$

The ground state is denoted in this paper by 


$$
\Omega_{\kappa}(x)=\frac{1}{(\pi \kappa)^{d / 4}} e^{-|x|^{2} / 2 \kappa} \equiv \phi_{s=0, \kappa}(x),
$$

where $x=\left(x_{1}, \ldots, x_{d}\right) \in \mathbb{R}^{d},|x|^{2}:=\sum_{j=1}^{d} x_{j}^{2}$.

Integral kernel of $G_{\kappa}(\beta)=e^{-\beta h_{\kappa}}$ has the explicit form (Mehler's formula for oscillator processes),

$$
G_{\kappa}(\beta ; x, y)=\frac{\exp \left\{-(2 \kappa)^{-1} \tanh (\beta / 2 \kappa)\left(|x|^{2}+|y|^{2}\right)-|x-y|^{2} /(2 \kappa \sinh (\beta / \kappa))\right\}}{\left\{\pi \kappa\left(1-e^{-2 \beta / \kappa}\right)\right\}^{d / 2}} .
$$

Here the operator $G_{\kappa}(\beta)$ belongs to the trace-class $\mathfrak{C}_{1}\left(L^{2}\left(\mathbb{R}^{d}\right)\right)$, with the trace norm equal to $\operatorname{Tr} G_{\kappa}(\beta)=1 /\left(1-e^{-\beta / \kappa}\right)^{d}=O\left(\kappa^{d}\right)$ for large $\kappa$. The largest eigenvalue of $G_{\kappa}(\beta)$ coincides with the operator norm $\left\|G_{\kappa}(\beta)\right\|=1$. We write all the eigenvalues of operator $G_{\kappa}(\beta)$ in decreasing order,

$$
g_{0}^{(\kappa)}=1>g_{1}^{(\kappa)}=e^{-\beta / \kappa} \geq g_{2}^{(\kappa)} \geq \cdots .
$$

The expectation value of total number of particles is given by

$$
N_{\kappa}(\beta, \mu)=\frac{1}{\beta} \frac{\partial \ln \Xi_{0, \kappa}(\beta, \mu)}{\partial \mu}=\sum_{s \in \mathbb{Z}_{+}^{d}} \frac{1}{e^{\beta\left(\epsilon_{\kappa}(s)-\mu\right)}-1} .
$$

Since value (1.10) diverges in the WHT limit $\kappa \rightarrow \infty$ as $\kappa^{d}$, one introduces the scaled quantity, ${ }^{3,14}$

$$
\rho_{\kappa}(\beta, \mu):=\frac{1}{\kappa^{d}} \sum_{s \in \mathbb{Z}_{+}^{d}} \frac{1}{e^{\beta\left(\epsilon_{\kappa}(s)-\mu\right)}-1},
$$

which is a Darboux-Riemann sum for the integral

$$
\rho(\beta, \mu)=\lim _{\kappa \rightarrow \infty} \rho_{\kappa}(\beta, \mu)=\int_{[0, \infty)^{d}} \frac{d p}{e^{\beta\left(|p|_{1}-\mu\right)}-1}=\sum_{s=1}^{\infty} \frac{e^{\beta \mu s}}{(\beta s)^{d}} .
$$

Since $\kappa^{d}$ may be interpreted as the effective "volume" (cf. Remark $\left.(1.1)\right), \rho(\beta, \mu)$ and $\rho_{\kappa}(\beta, \mu)$ are regarded as the expectation value of effective space-averaged density of the system, which has nonhomogeneous space distribution. One defines its critical value as usual,

$$
\rho_{c}(\beta):=\sup _{\mu<0} \rho(\beta, \mu)=\zeta(d) / \beta^{d} .
$$

Notice that (1.13) is bounded for $d>1$. Therefore, if $\rho>\rho_{c}(\beta)$, the IBG in the WHT limit $\kappa$ $\rightarrow \infty$ manifests a BEC in the scaled oscillator ground state (1.8) with the expected space-averaged condensate density,

$$
\rho_{0}(\beta):=\rho-\rho_{c}(\beta)=\lim _{\kappa \rightarrow \infty} \frac{1}{\kappa^{d}} \frac{1}{e^{-\beta \bar{\mu}_{\kappa}(\beta, \rho)}-1} .
$$

Moreover the expected local density $\rho_{0}(\beta)(x)$ can be defined such that

$$
\rho_{0}(\beta)(x)=\lim _{\kappa \rightarrow \infty} \frac{1}{\kappa^{d}} \frac{\Omega_{\kappa}(x)^{2}}{e^{-\beta \bar{\mu}_{\kappa}(\beta, \rho)}-1}
$$

holds. Here $\bar{\mu}_{\kappa}(\beta, \rho)$ is the unique root of the equation, $\rho=\rho_{\kappa}(\beta, \mu)$ [cf. (1.11)]. Their limits satisfy

$$
\lim _{\kappa \rightarrow \infty} \bar{\mu}_{\kappa}\left(\beta, \rho<\rho_{c}(\beta)\right)<0 \quad \text { and } \quad \lim _{\kappa \rightarrow \infty} \bar{\mu}_{\kappa}\left(\beta, \rho \geq \rho_{c}(\beta)\right)=0,
$$

especially 


$$
\bar{\mu}_{\kappa}\left(\beta, \rho>\rho_{c}(\beta)\right)=-\frac{1}{\beta\left(\rho-\rho_{c}(\beta)\right) \kappa^{d}}+o\left(\kappa^{-d}\right) .
$$

The integrated density of states $\mathcal{N}_{\kappa}(E)$ for the operator $h_{\kappa}$ is given by

$$
\mathcal{N}_{\kappa}(E)=\frac{1}{\kappa^{d}} \sum_{s \in \mathbb{Z}_{+}^{d}} \theta\left(E-|s|_{1} / \kappa\right) .
$$

Then from its Laplace transform

$$
\int_{0}^{\infty} e^{-t E} d \mathcal{N}_{\kappa}(E)=[\kappa(1-\exp (-t / \kappa))]^{-d}
$$

we obtain the $\kappa \rightarrow \infty$ limit

$$
d \mathcal{N}(E)=\frac{E^{d-1}}{\Gamma(d)} d E .
$$

In terms of this density of states, (1.11) and (1.12) are written as

$$
\rho_{\kappa}(\beta, \mu)=\int_{0}^{\infty} \frac{d \mathcal{N}_{\kappa}(E)}{e^{\beta(E-\mu)}-1}, \quad \rho(\beta, \mu)=\int_{0}^{\infty} \frac{d \mathcal{N}(E)}{e^{\beta(E-\mu)}-1} .
$$

It is instructive to compare these results with properties of the IBG "prepared" via standard thermodynamic limit $L \rightarrow \infty$ (1.3) for, e.g., Dirichlet boundary conditions. It is well known ${ }^{24}$ that in this case the expected boson density is

$$
\rho_{\Lambda_{L}}(\beta, \mu)=\frac{1}{\left|\Lambda_{L}\right|} \sum_{j \in \mathbb{Z}_{+}} \frac{1}{e^{\beta\left(\varepsilon_{L}(j)-\mu\right)}-1} .
$$

Here $\left\{\varepsilon_{L}(j)\right\}_{j \geq 0}$ is the spectrum of the one-particle operator $t_{L}$ and $\mu<\min _{j \geq 0} \varepsilon_{L}(j) \rightarrow 0$ as $L$ $\rightarrow \infty$. It also has the expression

$$
\rho_{\Lambda_{L}}(\beta, \mu)=\int_{0}^{\infty} \frac{d \tilde{\mathcal{N}}_{L}(E)}{e^{\beta(E-\mu)}-1}
$$

in terms of the integrated density of states $\left\{\tilde{\mathcal{N}}_{L}(E)\right\}_{L>0}$. The thermodynamic limit $\tilde{\mathcal{N}}(E)$ $=\lim _{L \rightarrow \infty} \tilde{\mathcal{N}}_{L}(E)$ is independent of "nonsticky" boundary conditions ${ }^{16}$ and is given by

$$
d \tilde{\mathcal{N}}(E)=\frac{E^{(d-2) / 2}}{(2 \pi)^{d / 2} \Gamma(d / 2)} d E .
$$

Then we get the limit of the expected density

$$
\widetilde{\rho}(\beta, \mu)=\lim _{L \rightarrow \infty} \rho_{\Lambda_{L}}(\beta, \mu)=\int_{0}^{\infty} \frac{d \tilde{\mathcal{N}}(E)}{e^{\beta(E-\mu)}-1} .
$$

The critical particle density for the IBG is

$$
\widetilde{\rho}_{c}(\beta):=\sup _{\mu<0} \widetilde{\rho}(\beta, \mu)=\int_{0}^{\infty} \frac{d \tilde{\mathcal{N}}(E)}{e^{\beta E}-1}=\zeta(d / 2) /(2 \pi \beta)^{d / 2} .
$$

Note the difference between $\tilde{\rho}_{c}(\beta)$ and $\rho_{c}(\beta)$. In particular, (1.23) is bounded only when $d>2$. Thus thermodynamic properties of the IBG in the standard TDL $\Lambda_{L} \rightarrow \mathbb{R}^{d}$ and the WHT limit $\kappa$ 
$\rightarrow \infty$ are different in spite of delusive impression that they have to produce identical systems.

Now we consider the mean-field interacting bosons trapped in the harmonic potential (1.1). Its grand-canonical partition function is given by

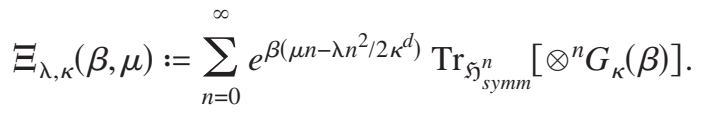

We consider the case of $\beta>0, \lambda>0$ and arbitrary $\mu \in \mathbb{R}$. Hereafter, we suppress the symbol $\lambda$ from the left-hand side of (1.24) since we fix $\lambda>0$ in the rest of the paper.

Remark 1.1: The scaling with the "volume" $\kappa^{d}$ (imposed by the WHT) is a conventional way to consider the Bose-Einstein condensation in traps, see Ref. 3, 10, and 14. Our definition of the mean-field (MF) interaction in WHT applies a space-average over the "volume" $\kappa^{d}$, which plays the same role as $\left|\Lambda_{L}\right|$ in the standard mean-field model where the interaction has the form $\lambda n^{2} / 2\left|\Lambda_{L}\right|$, see, e.g., Refs. 8 and 24.

Notice that $\lambda>0$ corresponds to repulsive MF particle interaction, whereas $\lambda=0$ is the case of the IBG (1.4).

In the present paper we consider in (1.24) only the "square" mean-field repulsive interaction $U_{2}:=\lambda n^{2} / 2 \kappa^{d}$. Although application of the large deviation technique makes it possible to consider also the case of the general MF interaction $U_{\Phi}:=\kappa^{d} \Phi\left(n / \kappa^{d}\right)$, where $\Phi: \mathbb{R} \rightarrow \mathbb{R}$ is a piecewise differentiable continuous function bounded from below.

To study the nonhomogeneous condensation and the space distribution of the constituent bosons in system (1.24), we use the RPF $\nu_{\kappa, \beta}$, i.e., the probability measure on the space of locally finite point measures with generating functional,

$$
E_{\kappa, \beta, \mu}(f):=\mathbb{E}_{\kappa, \beta, \mu}\left[e^{-\langle f, \xi\rangle}\right]=\frac{1}{\Xi_{\kappa}(\beta, \mu)} \sum_{n=0}^{\infty} e^{\beta \mu n-\beta \lambda n^{2} / 2 \kappa^{d}} \operatorname{Tr}_{\mathfrak{H}_{\text {symm }}^{n}}\left[\otimes^{n}\left(G_{\kappa}(\beta) e^{-f}\right)\right],
$$

where $f \in C_{0}\left(\mathbb{R}^{d}\right), f \geq 0$. Here $E_{\kappa, \beta, \mu}[\cdot]$ stands for expectation with respect to $\nu_{\kappa, \beta, \mu}(d \xi)$, and $\xi$ denotes the integral variable which represents locally finite point measure, see Refs. 1 and 18-20. The measure $\nu_{\kappa, \beta, \mu}$ describes a finite RPF whose Janossy measure can be given explicitly, see Remark 2.1.

In the present paper we study the properties of the MF interacting boson RPF $\nu_{\kappa, \beta, \mu}$ in the WHT limit $\kappa \rightarrow \infty$ by analyzing the generating functional (1.25). To this end we first define the MF critical chemical potential

$$
\mu_{\lambda, c}(\beta):=\lambda \int_{[0, \infty)^{d}} \frac{d p}{e^{\beta|p|_{1}}-1}=\lambda \int_{0}^{\infty} \frac{d \mathcal{N}(E)}{e^{\beta(E-\mu)}-1}=\frac{\lambda \zeta(d)}{\beta^{d}} .
$$

This critical parameter is similar to the critical chemical potential $\tilde{\mu}_{c}(\beta):=\lambda \widetilde{\rho}_{c}(\beta)$ for the standard homogeneous MF boson gas via TDL, see, e.g., Ref. 24 and (1.23).

\section{Main results}

Now we can formulate our main theorem.

Theorem 1.2: (i) Let $\mu<\mu_{\lambda, c}(\beta)$ (normal phase). Then the RPF $\nu_{\kappa, \beta, \mu}$ defined by (1.25), converges weakly in the WHT limit $\kappa \rightarrow \infty$ to the boson RPF $\nu_{\beta, r_{*}}$ corresponding to the generating functional,

$$
E_{\beta, r_{*}}(f):=\mathbb{E}_{\beta, r_{*}}\left[e^{-\langle f, \xi\rangle}\right]=\operatorname{det}\left[1+\sqrt{1-e^{-f}} r_{*} G(\beta)\left(1-r_{*} G(\beta)\right)^{-1} \sqrt{1-e^{-f}}\right]^{-1} .
$$

Here $E_{\beta, r_{*}}[\cdot]$ denotes expectation with respect to the measure $\nu_{\beta, r_{*}}$, det stands for the Fredholm determinant, $G(\beta):=e^{\beta \Delta / 2}$ is the heat semigroup on $\mathfrak{H}$, and $r_{*}=r_{*}(\beta, \mu, \lambda) \in(0,1)$ is a unique solution of the equation 


$$
\beta \mu=\log r+\lambda \beta \int_{0}^{\infty} \frac{d \mathcal{N}(E)}{r^{-1} e^{\beta E}-1} .
$$

(ii) For $\mu>\mu_{\lambda, c}(\beta)$ (condensed phase), the generating functional (1.25) has the following asymptotics:

$$
\lim _{\kappa \rightarrow \infty} \frac{1}{\kappa^{d / 2}} \log E_{\kappa, \beta, \mu}(f)=-\frac{\mu-\mu_{\lambda, c}(\beta)}{\pi^{d / 2} \lambda}\left(\sqrt{1-e^{-f}},\left(1+K_{f}\right)^{-1} \sqrt{1-e^{-f}}\right),
$$

where $K_{f}:=\left(G(\beta)^{1 / 2}(1-G(\beta))^{-1 / 2} \sqrt{1-e^{-f}}\right)^{*}\left(G(\beta)^{1 / 2}(1-G(\beta))^{-1 / 2} \sqrt{1-e^{-f}}\right)$ is a positive trace-class operator on $\mathfrak{H}=L^{2}\left(\mathbb{R}^{d}\right)$ for $d>2$.

Remark 1.3: For dimensions $d>1$ the integral (1.26) is finite. This gives an idea that the $B E C$ is possible for the case $\mu>\mu_{\lambda, c}(\beta)$ and dimensions $d>1$. However, in the present paper we assume $d>2$ to be able to prove our main theorem. In fact for $d=2$, the operator $K_{f}$ is not a trace-class operator and the Fredholm determinant is not well defined, see Ref. 19.

Remark 1.4: Because of a technical difficulty, actually we do not have results for the critical case $\mu=\mu_{\lambda, c}(\beta)$.

Remark 1.5: The heat semigroup $G(\beta)$ is appeared here since its kernel

$$
G(\beta ; x, y)=(2 \pi \beta)^{-d / 2} e^{-|x-y|^{2} / 2 \beta}
$$

is the pointwise limit of the Mehler kernel (1.9), as $\kappa \rightarrow \infty$. Therefore the generating functional of the resulting RPF (1.27) in (i) has exactly the same form as that for the standard homogeneous $I B G$ in the noncondensed phase, see Eq. (2.13) in Ref. 18, see also Ref. 17. That is, the position distribution of the model in the noncondensed phase is coincides with that of the standard IBG. However, it is the integrated density of states $\mathcal{N}(E)$ that is appeared in (1.28) instead of $\tilde{\mathcal{N}}(E)$. It implies that the dependence of $r_{*}$ as a function of parameters, especially $\beta$, is different from standard IBG or the standard MF model.

Remark 1.6: The RPF describing BEC for homogeneous IBG is given by the convolution of two RPFs. ${ }^{19}$ One convolution component is the usual boson RPF, while the other component seems to describe the position distribution of the condensed part of the constituent bosons. The behavior of the generating functional in case (ii) shows that the latter component overwhelms the former in the present model. It is to be noted here that the latter cannot be explained by the particles in the ground state alone, it contains the effect of the interference between "the condensed part" and "the normal part," although the intensity of the RPF is proportional to the square of the ground state wave function, as (1.15).

The sharp contrast between two regimes (i) and (ii) in Theorem 1.2 may be seen by the expectation values.

Corollary 1.7: For the case (i) $\mu<\mu_{\lambda, c}(\beta)$ (normal phase)

$$
\mathbb{E}_{\beta, r_{*}}[\langle f, \xi\rangle]=\operatorname{Tr}\left[f r_{*} G(\beta)\left(1-r_{*} G(\beta)\right)^{-1}\right]=\rho_{r_{*}} \int_{\mathbb{R}^{d}} f(x) d x
$$

holds, where $\rho_{r_{*}}$ is given by

$$
\rho_{r_{*}}=r_{*} G(\beta)\left(1-r_{*} G(\beta)\right)^{-1}(x, x)=\sum_{n=1}^{\infty} r_{*}^{n} /(2 \pi \beta n)^{d / 2} .
$$

For the case (ii) $\mu>\mu_{\lambda, c}(\beta)$ (condensed phase),

$$
\liminf _{\kappa \rightarrow \infty} \frac{E_{\kappa, \beta, \mu, \lambda}[\langle f, \xi\rangle]}{\kappa^{d / 2}} \geq \frac{\mu-\mu_{\lambda, c}(\beta)}{\pi^{d / 2} \lambda} \int_{\mathbb{R}^{d}} f(x) d x
$$

holds, where $f \in C_{0}\left(\mathbb{R}^{d}\right)$. 
The weak limits of the RPFs concerns the limit of the local position distribution of particles. In this sense, the results of Theorem 1.2 and Corollary 1.7 in regime (i) may be interpreted as follows: in the WHT limit the position distribution of the MF interacting bosons in neighborhoods of the origin of coordinates (i.e., the bottom of the WHT potential) is close to that of a free IBG corresponding to the unconventional parameter (1.28). The information about the particle position distribution in domains distant from the bottom of the WHT are missing in the limit $\nu_{\beta, r_{*}}$. In order to take this "tail" particles into account, we use the standard definition of the grand-canonical total number of particles for our model,

$$
\rho_{\kappa, \lambda}^{(\text {tot })}(\beta, \mu):=\frac{1}{\kappa^{d} \beta} \frac{\partial \ln \Xi_{\kappa}(\beta, \mu)}{\partial \mu}=\frac{1}{\kappa^{d} \Xi_{\kappa, \lambda}(\beta, \mu)} \sum_{n=0}^{\infty} n e^{\beta\left(\mu n-\lambda n^{2} / 2 \kappa^{d}\right)} \operatorname{Tr}_{\mathfrak{H}_{\mathrm{symm}}^{n}}\left[\otimes^{n} G_{\kappa}(\beta)\right] .
$$

Since $\kappa^{d}$ is interpreted as the effective volume of the model, $\rho_{\kappa, \lambda}^{(\text {tot })}(\beta, \mu)$ represents the effective total space-averaged density of the nonhomogeneous system (1.24).

Theorem 1.8: The WHT limit of (1.31)

$$
\rho_{\lambda}^{(\text {tot })}(\beta, \mu)=\lim _{\kappa \rightarrow \infty} \rho_{\kappa, \lambda}^{(\text {tot })}(\beta, \mu)=\lim _{\kappa \rightarrow \infty} \kappa^{-d} \operatorname{Tr}\left[r_{*} G_{\kappa}\left(1-r_{*} G_{\kappa}\right)^{-1}\right]
$$

exists and satisfies

(i)

$$
\begin{aligned}
& \left(\mu \leq \mu_{\lambda, c}(\beta)\right) \\
& \rho_{\lambda}^{(\mathrm{tot})}(\beta, \mu)=\int_{[0, \infty)^{d}} \frac{d \mathcal{N}(E)}{r_{*}^{-1} e^{\beta E}-1} \text { and } \beta \mu=\log r_{*}+\lambda \beta \rho_{\lambda}^{(\mathrm{tot})}(\beta, \mu),
\end{aligned}
$$

(ii) $\quad\left(\mu>\mu_{\lambda, c}(\beta)\right)$

$$
\rho_{\lambda}^{(\mathrm{tot})}(\beta, \mu)=\mu / \lambda
$$

It also holds that

$$
\rho_{c}^{(\mathrm{tot})}(\beta):=\lim _{\mu \rightarrow \mu_{c}(\beta)} \rho_{\lambda}^{(\mathrm{tot})}(\beta, \mu)=\zeta(d) / \beta^{d} .
$$

Remark 1.9: The readers should not to confuse two "densities:" $\rho_{r_{*}}$ in Corollary 1.7 and $\rho_{\lambda}^{\text {(tot) }}(\beta, \mu)$ defined above. The $\rho_{r_{*}}$ can be interpreted as the limit of the "local" density around the origin of coordinate of nonhomogeneous $R P F \nu_{\kappa, \beta, \mu}$; on the other hand the $\rho_{\lambda}^{\text {(tot) }}(\beta, \mu)$ retains the information about the expectation of the total number of particles with respect to $\nu_{\kappa, \beta, \mu}$ through the WHT limit.

Note that $\rho_{c}^{(\mathrm{tot})}(\beta)$ coincides with (1.13) of IBG in the WHT.

Remark 1.10: Qualitatively different behavior of the space distributions of bosons described in Theorem 1.2 can be understand heuristically with the help of Theorem 1.8 in the following way. Consider the WHT limit $\kappa \rightarrow \infty$.

In case (i) the bosons are distributed almost uniformly in the region of radius $\kappa$ according to kernel (1.9).

On the other hand, in case (ii) (condensed phase) the condensed part of particles $\kappa^{d}\left(\rho_{\lambda}^{(\mathrm{tot})}\right.$ $\left.\times(\beta, \mu)-\rho_{\lambda, c}^{\text {(tot) }}(\beta)\right)=\kappa^{d}\left(\mu-\mu_{\lambda, c}(\beta)\right) / \lambda$ is localized in the region of radius $O\left(\kappa^{1 / 2}\right)$ according to the profile of the square of the ground state wave function $\Omega_{\kappa}$. Whereas the particles outside of the condensate essentially spread out over the region of radius $\kappa$.

This paper is organized as follows. Preliminary estimates and results concerning the WHT limit for the mean-field interacting boson gas $(\lambda>0)$ are collected in Sec. II. Sections III and IV are dedicated to the proof of Theorems 1.2 and 1.8, respectively. We reserved Sec. V for summary and conjectures. 


\section{PRELIMINARY ARGUMENTS AND ESTIMATES}

In this section, we write expectation (1.25) as the ratio $\widetilde{\Xi}_{\kappa}(\beta, \mu) / \Xi_{\kappa}(\beta, \mu)$. The representations of $\widetilde{\Xi}_{\kappa}(\beta, \mu)$ and $\Xi_{\kappa}(\beta, \mu)$ are given in the form of integration of Fredholm determinants. We also give the miscellaneous estimates needed for the evaluation of these integrals.

\section{A. $\Xi_{\kappa}(\beta, \mu)$ and $\tilde{\Xi}_{\kappa}(\beta, \mu)$}

In terms of the projection operator on $\mathfrak{H}^{n}=\otimes^{n} L^{2}\left(\mathbb{R}^{d}\right)$ onto its subspace $\mathfrak{H}_{\text {symm }}^{n}$, the grandcanonical partition function can be written as

$$
\Xi_{\kappa}(\beta, \mu)=\sum_{n=0}^{\infty} e^{\beta \mu n-\beta \lambda n^{2} / 2 \kappa^{d}} \frac{1}{n !} \sum_{\sigma \in \mathcal{S}_{n}} \operatorname{Tr}_{\otimes^{n} L^{2}\left(\mathbb{R}^{d}\right)}\left[\left(\otimes^{n} G_{\kappa}(\beta)\right) U(\sigma)\right],
$$

where the second sum is taken over the symmetric group $\mathcal{S}_{n}$ and

$$
U(\sigma) \varphi_{1} \otimes \cdots \otimes \varphi_{n}=\varphi_{\sigma^{-1}(1)} \otimes \cdots \otimes \varphi_{\sigma^{-1}(n)} \quad \text { for } \quad \sigma \in \mathcal{S}_{n}, \varphi_{1}, \ldots, \varphi_{n} \in L^{2}\left(\mathbb{R}^{d}\right) .
$$

Hence we have

$$
\begin{aligned}
\Xi_{\kappa}(\beta, \mu) & =\sum_{n=0}^{\infty} \frac{e^{\beta \mu n-\beta \lambda n^{2} / 2 \kappa^{d}}}{n !} \sum_{\sigma \in \mathcal{S}_{n}} \int_{\left(\mathbb{R}^{d}\right)^{n}}\left(\prod_{j=1}^{n} G_{\kappa}(\beta)\left(x_{j}, x_{\sigma^{-1}(j)}\right)\right) d x_{1} \cdots d x_{n} \\
& =\sum_{n=0}^{\infty} \frac{e^{\beta \mu n-\beta \lambda n^{2} / 2 \kappa^{d}}}{n !} \int_{\left(\mathbb{R}^{d}\right)^{n}} \operatorname{per}\left\{G_{\kappa}(\beta)\left(x_{i}, x_{j}\right)\right\}_{1 \leq i, j \leq n} d x_{1} \cdots d x_{n},
\end{aligned}
$$

here per stands for the permanent of the matrix $\left\{G_{\kappa}(\beta)\left(x_{i}, x_{j}\right)\right\}_{1 \leq i, j \leq n}$.

Remark 2.1: The point field $\nu_{\kappa, \beta, \mu}$ of (1.25) can also be defined in terms of Janossy measures or exclusion probability. ${ }^{1}$ This means that $\nu_{\kappa, \beta, \mu}$ is a finite point field, which assigns the probability

$$
\operatorname{Pr}\left\{d X_{n}\right\}:=\frac{e^{\beta \mu n-\beta \lambda n^{2} / 2 \kappa^{d}}}{n ! \Xi_{\kappa}(\beta, \mu)} \operatorname{per}\left\{G_{\kappa}(\beta)\left(x_{i}, x_{j}\right)\right\}_{1 \leq i, j \leq n} d x_{1} \cdots d x_{n}
$$

to the event $\left\{d X_{n}\right\}$ : there are exactly $n$ points, one in each infinitesimal region $\prod_{i=1}^{d}\left(x_{j}^{(i)}, x_{j}^{(i)}\right.$ $\left.+d x_{j}^{(i)}\right), \quad\left(x_{j}=\left(x_{j}^{(1)}, \cdots, x_{j}^{(d)}\right), j=1, \cdots, n\right)$.

As in Refs. 18 and 19, we use the generalized Vere-Jones' formula ${ }^{17,22}$ in the form

$$
\frac{1}{n !} \int \operatorname{per}\left\{J\left(x_{i}, x_{j}\right)\right\}_{1 \leq i, j \leq n} d x_{1} \cdots d x_{n}=\oint_{S_{r}(0)} \frac{d z}{2 \pi i z^{n+1} \operatorname{det}(1-z J)},
$$

where $r>0$ satisfies $\|r J\|<1 . S_{r}(\zeta)$ denotes the integration contour defined by the map $\theta \mapsto \zeta$ $+r \exp (i \theta)$, where $\theta$ ranges from $-\pi$ to $\pi, r>0$, and $\zeta \in \mathrm{C}$. Then we obtain

$$
\Xi_{\kappa}(\beta, \mu)=\sum_{n=0}^{\infty} e^{\beta \mu n-\beta \lambda n^{2} / 2 \kappa^{d}} \oint_{S_{r}(0)} \frac{d z}{2 \pi i z^{n+1} \operatorname{det}\left(1-z G_{\kappa}(\beta)\right)},
$$

where $r \in\left(0,\left\|G_{\kappa}(\beta)\right\|^{-1}\right)=(0,1)$. Note that the zeroth term is 1 in this expression.

Let us substitute

$$
e^{-\beta \lambda n^{2} / 2 \kappa^{d}}=\sqrt{\frac{\beta \lambda}{2 \pi \kappa^{d}}} \int_{\mathbb{R}} d x \exp \left(-\frac{\beta \lambda}{2 \kappa^{d}}\left((x+i s)^{2}-2 i n(x+i s)\right)\right) .
$$

If $s>0$ satisfies 


$$
e^{\beta \mu-\beta \lambda s / \kappa^{d}}<r,
$$

we can take the summation over $n$ together with the complex integration and a scaling of $x$ to get

$$
\begin{aligned}
\Xi_{\kappa}(\beta, \mu) & =\sqrt{\frac{\beta \lambda}{2 \pi \kappa^{d}}} e^{\beta \lambda s^{2} / 2 \kappa^{d}} \int_{\mathbb{R}} d x \oint \frac{d z}{2 \pi i} \frac{e^{-\beta \lambda\left(x^{2}+2 i s x\right) / 2 \kappa^{d}}}{\left(z-e^{\beta \mu+\beta \lambda(i x-s) / \kappa^{d}}\right) \operatorname{Det}\left[1-z G_{\kappa}(\beta)\right]} \\
& =\sqrt{\frac{\kappa^{d}}{2 \pi \beta \lambda}} e^{\beta \lambda s^{2} / 2 \kappa^{d}} \int_{\mathbb{R}} d x \frac{e^{-i s x-\kappa^{d} x^{2} / 2 \beta \lambda}}{\operatorname{Det}\left[1-e^{\beta \mu+i x-\beta \lambda s / \kappa^{d}} G_{\kappa}(\beta)\right]} .
\end{aligned}
$$

Note that after $z$-integration, $r$ disappears and (2.2) is valid for any $s$ satisfying $\exp (\beta \mu$ $\left.-\beta \lambda s / \kappa^{d}\right) \in\left(0,\left\|G_{\kappa}(\beta)\right\|^{-1}\right)$. We will estimate the integral in the spirit of saddle point method. Here, we extract the main part from the integral. Let $s=s_{\kappa}, r=r_{\kappa}$ be the unique solution of the system

$$
\begin{gathered}
r=\exp \left(\beta \mu-\beta \lambda s / \kappa^{d}\right), \\
s=\operatorname{Tr}\left[r G_{\kappa}(\beta)\left(1-r G_{\kappa}(\beta)\right)^{-1}\right] .
\end{gathered}
$$

Obviously, the condition $r_{\kappa} \in\left(0,\left\|G_{\kappa}(\beta)\right\|^{-1}\right)$ is fulfilled. Hence, we can substitute in (2.2) $s$ by $s_{\kappa}$. Using the product property of the Fredholm determinant, we get for denominator of (1.25) the representation,

$$
\Xi_{\kappa}(\beta, \mu)=\sqrt{\frac{\kappa^{d}}{2 \pi \beta \lambda}} \frac{e^{\beta \lambda s_{\kappa}^{2} / 2 \kappa^{d}}}{\operatorname{Det}\left[1-r_{\kappa} G_{\kappa}(\beta)\right]} \int_{\mathbb{R}} d x \frac{e^{-i s_{\kappa} x-\kappa^{d} x^{2} / 2 \beta \lambda}}{\operatorname{det}\left[1-\left(e^{i x}-1\right) r_{\kappa} G_{\kappa}(\beta)\left(1-r_{\kappa} G_{\kappa}(\beta)\right)^{-1}\right]} .
$$

For the numerator of (1.25), we introduce bounded symmetric operators

$$
\tilde{G}_{\kappa}(\beta)(f):=G_{\kappa}(\beta)^{1 / 2} e^{-f} G_{\kappa}(\beta)^{1 / 2},
$$

indexed by function $f \in C_{0}\left(\mathbb{R}^{d}\right), f \geq 0$, which we skip below for simplicity. Then for generating functional (1.25) one gets the form: $E_{\kappa, \beta, \mu}(f)=\widetilde{\Xi}_{\kappa}(\beta, \mu)(f) / \Xi_{\kappa}(\beta, \mu)$, where

$$
\begin{aligned}
\widetilde{\Xi}_{\kappa}(\beta, \mu)(f) & =\widetilde{\Xi}_{\kappa}(\beta, \mu)=\sum_{n=0}^{\infty} e^{\beta \mu n-\beta \lambda n^{2} / 2 \kappa^{d}} \operatorname{Tr}_{\mathfrak{H}_{\mathrm{symm}}^{n}}\left[\otimes^{n} \widetilde{G}_{\kappa}(\beta)\right] \\
& =\sqrt{\frac{\kappa^{d}}{2 \pi \beta \lambda}} \frac{e^{\beta \lambda \tilde{s}_{\kappa}^{2} / 2 \kappa^{d}}}{\operatorname{det}\left[1-\widetilde{r}_{\kappa} \widetilde{G}_{\kappa}(\beta)\right]} \int_{\mathrm{R}} d x \frac{e^{-i \tilde{s}_{\kappa} x-\kappa^{d} x^{2} / 2 \beta \lambda}}{\operatorname{det}\left[1-\left(e^{i x}-1\right) \widetilde{r}_{\kappa} \widetilde{G}_{\kappa}(\beta)\left(1-\widetilde{r}_{\kappa} \widetilde{G}_{\kappa}(\beta)\right)^{-1}\right]} .
\end{aligned}
$$

Here $\left(\tilde{s}_{\kappa}, \tilde{r}_{\kappa}\right)$ is the unique solution of

$$
\begin{gathered}
\tilde{r}=\exp \left(\beta \mu-\beta \lambda \widetilde{s} / \kappa^{d}\right) \\
\tilde{s}=\operatorname{Tr}\left[\tilde{r} \widetilde{G}_{\kappa}(\beta)\left(1-\tilde{r} \widetilde{G}_{\kappa}(\beta)\right)^{-1}\right] .
\end{gathered}
$$

Obviously, $\widetilde{r}_{\kappa} \in\left(0,\left\|\widetilde{G}_{\kappa}(\beta)\right\|^{-1}\right)$. Note also that $r_{\kappa}$ and $\widetilde{r}_{\kappa}$ satisfy the following conditions, respectively,

$$
\frac{1}{\kappa^{d}} \operatorname{Tr}\left[r_{\kappa} G_{\kappa}(\beta)\left(1-r_{\kappa} G_{\kappa}(\beta)\right)^{-1}\right]=\frac{\beta \mu-\log r_{\kappa}}{\beta \lambda}
$$




$$
\frac{1}{\kappa^{d}} \operatorname{Tr}\left[\widetilde{r}_{\kappa} \widetilde{G}_{\kappa}(\beta)\left(1-\widetilde{r}_{\kappa} \widetilde{G}_{\kappa}(\beta)\right)^{-1}\right]=\frac{\beta \mu-\log \widetilde{r}_{\kappa}}{\beta \lambda} .
$$

Since by definition (2.5) one obviously gets $\widetilde{G}_{\kappa}(\beta) \leq G_{\kappa}(\beta)$, the operator $\widetilde{G}_{\kappa}(\beta)$ also belongs to the trace class $\mathcal{C}_{1}(\mathfrak{H})$. We put the eigenvalues of $\widetilde{G}_{\kappa}(\beta)$ in the decreasing order

$$
\widetilde{g}_{0}^{(\kappa)}=\left\|\widetilde{G}_{\kappa}(\beta)\right\| \geq \widetilde{g}_{1}^{(\kappa)} \geq \cdots .
$$

Then, we have $g_{j}^{(\kappa)} \geq \widetilde{g}_{j}^{(\kappa)}(j=0,1,2, \ldots)$ by the min-max principle.

\section{B. Approximations of one-particle Gibbs semigroups}

Here we establish some relations between Gibbs semigroup $\left\{G_{\kappa}(\beta)\right\}_{\beta \geq 0}$ and the heat semigroup $\{G(\beta)\}_{\beta \geq 0}$. Let $P_{\kappa}$ be the orthogonal projection on $\mathfrak{H}$ onto its one-dimensional subspace spanned by the vector $\Omega_{\kappa}$, and put $Q_{\kappa}:=I-P_{\kappa}$.

Lemma 2.2: For any $r \in(0,1)$,

$$
\begin{gathered}
\left\|\sqrt{1-e^{-f}}\left[r G_{\kappa}(\beta)\left(1-r G_{\kappa}(\beta)\right)^{-1}-r G(\beta)(1-r G(\beta))^{-1}\right] \sqrt{1-e^{-f}}\right\|_{1} \rightarrow 0, \\
\left\|\sqrt{1-e^{-f}} Q_{\kappa} G_{\kappa}(\beta) Q_{\kappa}\left(1-Q_{\kappa} G_{\kappa}(\beta) Q_{\kappa}\right)^{-1} \sqrt{1-e^{-f}}-K_{f}\right\|_{1} \rightarrow 0
\end{gathered}
$$

hold in the limit $\kappa \rightarrow \infty$, where $\|\cdot\|_{1}$ stands for the trace norm in $\mathcal{C}_{1}\left(L^{2}\left(\mathbb{R}^{d}\right)\right)$.

Proof: First, we show the estimates

$$
\begin{gathered}
\left|G_{\kappa}^{n}(\beta ; x, y)-G^{n}(\beta ; x, y)\right| \leq \frac{A^{\prime}}{\kappa n^{d / 2-1}}\left(1+\frac{|x|^{2}+|y|^{2}}{\kappa}\right) \quad \text { if } n \beta / \kappa \leq 1, \\
\left|G_{\kappa}^{n}(\beta ; x, y)-\Omega_{\kappa}(x) \Omega_{\kappa}(y)\right| \leq \frac{B^{\prime} e^{-n \beta / 2 \kappa}}{\kappa^{d / 2}}\left(1+\frac{|x-y|^{2}}{\kappa}\right) \quad \text { if } n \beta / \kappa \geq 1,
\end{gathered}
$$

where $A^{\prime}$ and $B^{\prime}$ depend only on $d$ and $\beta$. In fact, by Mehler's formula one gets for $n \beta / \kappa \leq 1$ the estimate

$$
\begin{aligned}
& \left|G_{\kappa}^{n}(\beta ; x, y)-G^{n}(\beta ; x, y)\right| \\
& \quad \leq \frac{1}{(2 \pi n \beta)^{d / 2}}\left|\left(\frac{2 n \beta / \kappa}{1-e^{-2 n \beta / \kappa}}\right)^{d / 2}-1\right| \exp \left(-\frac{\tanh (n \beta / 2 \kappa)}{2 \kappa}\left(|x|^{2}+|y|^{2}\right)-\frac{|x-y|^{2}}{2 \kappa \sinh (n \beta / \kappa)}\right) \\
& \quad+\frac{1}{(2 \pi n \beta)^{d / 2}}\left|\exp \left(-\frac{\tanh (n \beta / 2 \kappa)}{2 \kappa}\left(|x|^{2}+|y|^{2}\right)\right)-1\right| \exp \left(-\frac{|x-y|^{2}}{2 \kappa \sinh (n \beta / \kappa)}\right) \\
& \quad+\frac{1}{(2 \pi n \beta)^{d / 2}}\left|1-\exp \left(-\left(\frac{\sinh (n \beta / \kappa)}{(n \beta / \kappa)}-1\right) \frac{|x-y|^{2}}{2 \kappa \sinh (n \beta / \kappa)}\right)\right| \exp \left(-\frac{|x-y|^{2}}{2 \kappa \sinh (n \beta / \kappa)}\right) \\
& \quad \leq \frac{1}{(2 \pi n \beta)^{d / 2}}\left(\frac{A n \beta}{\kappa}+\frac{|x|^{2}+|y|^{2}}{2 \kappa} \frac{n \beta}{2 \kappa}+\left(\frac{n \beta}{\kappa}\right)^{2} \frac{|x-y|^{2}}{2 \kappa \sinh (n \beta / \kappa)}\right) \exp \left(-\frac{|x-y|^{2}}{2 \kappa \sinh (n \beta / \kappa)}\right) \\
& \quad \leq \frac{A^{\prime}}{\kappa n^{d / 2-1}}\left(1+\frac{|x|^{2}+|y|^{2}}{\kappa}\right) .
\end{aligned}
$$

Here we have used (A2) for the first term, (A4) and (A6) for the second term, and (A4) and (A8) for the third term at the second inequality and (A8) at the last inequality.

On the other hand, for $n \beta / \kappa \geq 1$, we obtain 


$$
\begin{aligned}
& \left|G_{\kappa}^{n}(\beta ; x, y)-\Omega_{\kappa}(x) \Omega_{\kappa}(y)\right| \\
& \quad \leq \frac{1}{(\pi \kappa)^{d / 2}}\left|\left(\frac{1}{1-e^{-2 n \beta / \kappa}}\right)^{d / 2}-1\right| \exp \left(-\frac{\tanh (n \beta / 2 \kappa)}{2 \kappa}\left(|x|^{2}+|y|^{2}\right)-\frac{|x-y|^{2}}{2 \kappa \sinh (n \beta / \kappa)}\right) \\
& \quad+\frac{1}{(\pi \kappa)^{d / 2}}\left|\exp \left(-\frac{\tanh (n \beta / 2 \kappa)}{2 \kappa}\left(|x|^{2}+|y|^{2}\right)\right)-\exp \left(-\frac{|x|^{2}+|y|^{2}}{2 \kappa}\right)\right| \exp \left(-\frac{|x-y|^{2}}{2 \kappa \sinh (n \beta / \kappa)}\right) \\
& \quad+\frac{1}{(\pi \kappa)^{d / 2}} \exp \left(-\frac{|x|^{2}+|y|^{2}}{2 \kappa}\right)\left|\exp \left(-\frac{|x-y|^{2}}{2 \kappa \sinh (n \beta / \kappa)}\right)-1\right| \\
& \quad \leq \frac{1}{(\pi \kappa)^{d / 2}}\left(B e^{-2 n \beta / \kappa}+\frac{1}{e}\left(\operatorname{coth}\left(\frac{n \beta}{2 \kappa}\right)-1\right)+\frac{|x-y|^{2}}{2 \kappa \sinh (n \beta / \kappa)}\right) \\
& \quad \leq \frac{B^{\prime} e^{-n \beta / 2 \kappa}}{\kappa^{d / 2}}\left(1+\frac{|x-y|^{2}}{\kappa}\right),
\end{aligned}
$$

where we have used (A3) for the first term, (A5) and (A7) for the second term, and (A4) and (A9) for the third term.

Now, let us show the second limit (2.11), notice that the inequality

$$
\begin{aligned}
& \sum_{n=1}^{\infty}\left|G_{\kappa}^{n}(\beta ; x, y)-G^{n}(\beta ; x, y)-\Omega_{0}^{\kappa}(x) \Omega_{0, \kappa}(y)\right| \\
& \leq \sum_{n=1}^{\lceil\kappa / \beta\rceil}\left(\frac{A^{\prime}}{\kappa n^{d / 2-1}}\left(1+\frac{|x|^{2}+|y|^{2}}{\kappa}\right)+\frac{1}{(\pi \kappa)^{d / 2}}\right) \\
& \quad+\sum_{n=[\kappa / \beta]+1}^{\infty}\left(\frac{B^{\prime} e^{-n \beta / 2 \kappa}}{\kappa^{d / 2}}\left(1+\frac{|x-y|^{2}}{\kappa}\right)+\frac{1}{(2 \pi n \beta)^{d / 2}}\right) \\
& \leq C^{\prime}\left(\kappa^{1-d / 2} \vee \kappa^{-1} \log \kappa\right)\left(1+\frac{|x|^{2}+|y|^{2}}{\kappa}\right)
\end{aligned}
$$

holds for $C^{\prime}$, which depends only on $d$ and $\beta$. The integer part is denoted by [·]. Here we used the estimates (2.12), (2.13) and

$$
\sum_{n=1}^{N} \frac{1}{n^{d / 2-1}}= \begin{cases}O(1) & (d / 2>2) \\ O\left(N^{2-d / 2}\right) & (1<) d / 2<2 \\ O(\log N) & d / 2=2\end{cases}
$$

and so on. Now put

$$
A^{(\kappa)}:=\sqrt{1-e^{-f}} Q_{\kappa} G_{\kappa}(\beta) Q_{\kappa}\left(1-Q_{\kappa} G_{\kappa}(\beta) Q_{\kappa}\right)^{-1} \sqrt{1-e^{-f}} .
$$

Then, since $\left\|Q_{\kappa} G_{\kappa}(\beta) Q_{\kappa}\right\|=e^{-\beta / \kappa}<1$, one gets in the limit $N \rightarrow \infty$ the operator-norm convergence

$$
A_{N}^{(\kappa)}=\sqrt{1-e^{-f}} \sum_{n=1}^{N} Q_{\kappa} G_{\kappa}(\beta)^{n} Q_{\kappa} \sqrt{1-e^{-f}} \rightarrow A^{(\kappa)} \geq 0 .
$$

Recall that Theorem 3.1(i) and Proposition 2.3(i) in Ref. 19 yield the strong convergence,

$$
K_{N}=\sqrt{1-e^{-f}} \sum_{n=1}^{N} G(\beta)^{n} \sqrt{1-e^{-f}} \rightarrow K_{f} \geq 0
$$

for $N \rightarrow \infty$. Moreover, we also have the following estimate for the operator norm. 


$$
\begin{aligned}
\left\|A_{N}^{(\kappa)}-K_{N}\right\|= & \sup _{\|\phi\|=1}\left|\left(\phi, \sqrt{1-e^{-f}} \sum_{n=1}^{N}\left(Q_{\kappa} G_{\kappa}(\beta)^{n} Q_{\kappa}-G(\beta)^{n}\right) \sqrt{1-e^{-f}} \phi\right)\right| \\
\leq & \sup _{\|\phi\|=1} \int_{\operatorname{supp} f} d x \int_{\operatorname{supp} f} d y \sqrt{1-e^{-f(x)}} \sqrt{1-e^{-f(y)}}|\phi(x) \phi(y)| \\
& \times \sum_{n=1}^{N}\left|G_{\kappa}^{n}(\beta ; x, y)-G^{n}(\beta ; x, y)-\Omega_{\kappa}(x) \Omega_{\kappa}(y)\right| \\
\leq & \left\|\sqrt{1-e^{-f}}\right\|^{2} \sup _{x, y \in \operatorname{supp} f} \sum_{n=1}^{\infty}\left|G_{\kappa}^{n}(\beta ; x, y)-G^{n}(\beta ; x, y)-\Omega_{0}^{\kappa}(x) \Omega_{0, \kappa}(y)\right| \rightarrow 0
\end{aligned}
$$

for $\kappa \rightarrow \infty$ uniformly in $N$. Here $\left\|\sqrt{1-e^{-f}}\right\|$ stands for the $L^{2}$ norm of the function. We have used Cauchy-Schwarz inequality at the second inequality. The standard $3 \epsilon$-argument yields that $A^{(\kappa)}$ $\rightarrow K_{f}$ strongly when $\kappa \rightarrow \infty$.

On the other hand, since the operators $A^{(\kappa)}, K_{f}$ are non-negative, we have for $\kappa \rightarrow \infty$ the limit

$$
\begin{aligned}
\left\|A^{(\kappa)}\right\|_{1}-\left\|K_{f}\right\|_{1} & =\operatorname{Tr} A^{(\kappa)}-\operatorname{Tr} K_{f} \\
& =\sum_{n=1}^{\infty}\left(\phi_{n},\left(A^{(\kappa)}-K_{f}\right) \phi_{n}\right) \\
& =\sum_{n=1}^{\infty} \sum_{l=1}^{\infty}\left(\phi_{n}, \sqrt{1-e^{-f}}\left(Q_{\kappa} G_{\kappa}(\beta)^{l} Q_{\kappa}-G(\beta)^{l}\right) \sqrt{1-e^{-f}} \phi_{n}\right) \\
& =\sum_{l=1}^{\infty} \sum_{n=1}^{\infty}\left(\phi_{n}, \sqrt{1-e^{-f}}\left(Q_{\kappa} G_{\kappa}(\beta)^{l} Q_{\kappa}-G(\beta)^{l}\right) \sqrt{1-e^{-f}} \phi_{n}\right) \\
& =\sum_{l=1}^{\infty} \operatorname{Tr} \sqrt{1-e^{-f}}\left(Q_{\kappa} G_{\kappa}(\beta)^{l} Q_{\kappa}-G(\beta)^{l}\right) \sqrt{1-e^{-f}} \\
& =\sum_{l=1}^{\infty} \int_{\operatorname{supp} f}\left(1-e^{-f(x)}\right)\left(G_{\kappa}^{l}(\beta ; x, x)-G^{l}(\beta ; x, x)-\Omega_{\kappa}(x)^{2}\right) d x \rightarrow 0,
\end{aligned}
$$

where $\left\{\phi_{n}\right\}_{n=1}^{\infty}$ is an arbitrary complete orthonormal system in $\mathfrak{H}$. Note that we can exchange the order of summations over $n$ and $l$ since

$$
\operatorname{Tr} A^{(\kappa)}=\sum_{n, l}\left(\phi_{n}, \sqrt{1-e^{-f}} Q_{\kappa} G_{\kappa}(\beta)^{l} Q_{\kappa} \sqrt{1-e^{-f}} \phi_{n}\right)
$$

and

$$
\operatorname{Tr} K_{f}=\sum_{n, l}\left(\phi_{n}, \sqrt{1-e^{-f}} G^{l}(\beta) \sqrt{1-e^{-f}} \phi_{n}\right)
$$

are convergent non-negative sequences. Here we used (2.14) and (2.15) in the third equality above. Thus, we get $\lim _{\kappa \rightarrow \infty} A^{(\kappa)}=K_{f}$ in $\mathfrak{C}_{1}(\mathfrak{H})$ by the Grümm convergence theorem, see, e.g., Ref. 23.

Let us consider the first limit (2.10). Since the identity $r G_{\kappa}(\beta)\left(1-r G_{\kappa}(\beta)\right)^{-1}=\sum_{n=1}^{\infty} r^{n} G_{\kappa}(\beta)^{n}$ holds in the operator-norm topology and $G_{\kappa}^{n}(\beta ; x, y) \geq 0$ [by virtue of Lemma 2.2(ii) in Ref. 19], we get the representation 


$$
\left[r G_{\kappa}(\beta)\left(1-r G_{\kappa}(\beta)\right)^{-1}\right](x, y)=\sum_{n=1}^{\infty} r^{n} G_{\kappa}^{n}(\beta ; x, y) .
$$

Similarly, one gets the representation

$$
\left[r G(\beta)(1-r G(\beta))^{-1}\right](x, y)=\sum_{n=1}^{\infty} r^{n} G^{n}(\beta ; x, y) .
$$

In fact, the series in the right-hand side of the above representations are pointwise convergent because the uniform estimates

$$
G_{\kappa}^{n}(\beta ; x, y) \leq\left(\frac{1}{\pi \beta} \vee \frac{2}{\pi \kappa}\right)^{d / 2}, \quad G^{n}(\beta ; x, y) \leq\left(\frac{1}{2 \pi \beta}\right)^{d / 2}
$$

hold for all $\kappa>0, x, y \in \mathbb{R}^{d}$, and $n \in \mathbb{N}$. Here we used (A1) for the first inequality. Hence we obtain the estimate

$$
\begin{aligned}
& \left|\left[r G_{\kappa}(\beta)\left(1-r G_{\kappa}(\beta)\right)^{-1}\right](x, y)-\left[r G(\beta)(1-r G(\beta))^{-1}\right](x, y)\right| \leq \sum_{n=1}^{\infty} r^{n}\left|G_{\kappa}^{n}(\beta ; x, y)-G^{n}(\beta ; x, y)\right| \\
& \quad \leq \sum_{n=1}^{\lceil\kappa / \beta \mid} r^{n} \frac{A^{\prime}}{\kappa n^{d / 2-1}}\left(1+\frac{|x|^{2}+|y|^{2}}{\kappa}\right)+\sum_{n=\{\kappa / \beta \mid+1}^{\infty} r^{n}\left[\left(\frac{1}{2 \pi \beta}\right)^{d / 2}+\left(\frac{1}{\pi \beta} \vee \frac{2}{\pi \kappa}\right)^{d / 2}\right] \\
& \quad \leq \frac{A^{\prime}}{\kappa(1-r)}\left(1+\frac{|x|^{2}+|y|^{2}}{\kappa}\right)+C \frac{r^{[\kappa / \beta \mid+1}}{1-r},
\end{aligned}
$$

which tends to zero when $\kappa \rightarrow \infty$, uniformly in $x, y \in \mathfrak{C}$ for any compact set $\mathfrak{C}$, where $C$ denotes a constant which depends only on $d$ and on $\beta$.

Thus,

$$
\sup _{x, y \in \operatorname{supp} f}\left|\left[r G_{\kappa}(\beta)\left(1-r G_{\kappa}(\beta)\right)^{-1}\right](x, y)-\left[r G(\beta)(1-r G(\beta))^{-1}\right](x, y)\right| \rightarrow 0
$$

holds. We get the first of the announced limits (2.10) by the similar (even simpler) argument to the second one. This finishes the proof of the lemma.

\section{Estimates for the scaled mean-field interaction}

In the following, we use the notation $B_{\kappa}:=\hat{O}\left(\kappa^{\alpha}\right)$, which means that there exist two numbers $c_{1} \geq c_{2}>0$ such that

$$
c_{1} \kappa^{\alpha} \geq B_{\kappa} \geq c_{2} \kappa^{\alpha} .
$$

Next, we put $W_{\kappa}:=\left(G_{\kappa}(\beta)\right)^{1 / 2} \sqrt{1-e^{-f}}$ and define $D_{\kappa}:=G_{\kappa}(\beta)-\widetilde{G}_{\kappa}(\beta)=W_{\kappa} W_{\kappa}^{*}$.

Lemma 2.3: For large $\kappa>0$, the following asymptotics hold

$$
\begin{gathered}
\left(\Omega_{\kappa}, D_{\kappa} \Omega_{\kappa}\right)=\left\|W_{\kappa}^{*} \Omega_{\kappa}\right\|^{2}=\left\|\sqrt{1-e^{-f}}\right\|^{2}(1+o(1)) /(\pi \kappa)^{d / 2}, \\
\operatorname{Tr} D_{\kappa}=\frac{\left\|\sqrt{1-e^{-f}}\right\|^{2}(1+o(1))}{\left(\pi \kappa\left(1-e^{-2 \beta / \kappa}\right)\right)^{d / 2}}
\end{gathered}
$$

and 


$$
\begin{aligned}
g_{0}^{(\kappa)}-\widetilde{g}_{0}^{(\kappa)} & =\left(\Omega_{\kappa},\left(D_{\kappa}-D_{\kappa} Q_{\kappa}\left[\widetilde{g}_{0}^{(\kappa)}-Q_{\kappa} \widetilde{G}_{\kappa}(\beta) Q_{\kappa}\right]^{-1} Q_{\kappa} D_{\kappa}\right) \Omega_{\kappa}\right) \\
& =\frac{1+o(1)}{(\pi \kappa)^{d / 2}}\left(\sqrt{1-e^{-f}},\left[1+W_{\kappa}^{*} Q_{\kappa}\left[1-Q_{\kappa} G_{\kappa}(\beta) Q_{\kappa}\right]^{-1} Q_{\kappa} W_{\kappa}\right]^{-1} \sqrt{1-e^{-f}}\right) \\
& =\hat{O}\left(\kappa^{-d / 2}\right) .
\end{aligned}
$$

Proof: For simplicity of notation we suppress everywhere below the index $\kappa$ in $g_{j}^{(\kappa)}, \widetilde{g}_{j}^{(\kappa)}, \Omega_{(\kappa)}$ and in $Q_{\kappa}$.

Note that the first equality is a straightforward consequence of definitions (1.8) and (2.5). The second equality can be derived directly from Mehler's formula.

Now by the min-max principle, for $d>2$ and $\kappa$ large enough, we obtain from the value $g_{1}$ $=\exp (-\beta / \kappa)$ the following estimates:

$$
g_{0}=1 \geq \widetilde{g}_{0} \geq\left(\Omega, \widetilde{G}_{\kappa}(\beta) \Omega\right)=1-\left(\Omega, D_{\kappa} \Omega\right)=1-\hat{O}\left(\kappa^{-d / 2}\right)>g_{1}=1-\hat{O}\left(\kappa^{-1}\right) \geq \widetilde{g}_{1} .
$$

Hence the eigenspace of the operator $\widetilde{G}_{\kappa}(\beta)$ for its largest eigenvalue $\widetilde{g}_{0}$ is one dimensional. Let $\widetilde{\Omega}$ be the normalized eigenfunction corresponding to $\widetilde{g}_{0}$ and let $\widetilde{\Omega}=a \Omega+\Omega^{\prime}$, with $\left(\Omega, \Omega^{\prime}\right)=0$. Then $\widetilde{G}_{\kappa}(\beta) \widetilde{\Omega}=\widetilde{g}_{0} \widetilde{\Omega}$ yields

$$
a \widetilde{G}_{\kappa}(\beta) \Omega+\widetilde{G}_{\kappa}(\beta) \Omega^{\prime}=a \widetilde{g}_{0} \Omega+\widetilde{g}_{0} \Omega^{\prime} .
$$

Applying to this relation orthogonal projector $P$ (on $\Omega$ ) and $Q=I-P$, we obtain

$$
\begin{gathered}
a g_{0}-a\left(\Omega, D_{\kappa} \Omega\right)-\left(\Omega, D_{\kappa} \Omega^{\prime}\right)=a \widetilde{g}_{0}, \\
-a Q D_{\kappa} \Omega+Q \widetilde{G}_{\kappa}(\beta) Q \Omega^{\prime}=\widetilde{g}_{0} \Omega^{\prime} .
\end{gathered}
$$

Since $Q \widetilde{G}_{\kappa}(\beta) Q \leq Q G_{\kappa}(\beta) Q \leq g_{1}<\widetilde{g}_{0}$, the operator $\widetilde{g}_{0}-Q \widetilde{G}_{\kappa}(\beta) Q$ is positive and invertible. It follows from the second identity that

$$
\Omega^{\prime}=-a\left[\widetilde{g}_{0}-Q \widetilde{G}_{\kappa}(\beta) Q\right]^{-1} Q D_{\kappa} \Omega .
$$

Together with the above first identity

$$
\begin{aligned}
g_{0}-\widetilde{g}_{0}= & \left(\Omega,\left(D_{\kappa}-D_{\kappa} Q\left[\widetilde{g}_{0}-Q \widetilde{G}_{\kappa}(\beta) Q\right]^{-1} Q D_{\kappa}\right) \Omega\right)=\left(W_{\kappa}^{*} \Omega,\left(1-W_{\kappa}^{*} Q\left[\widetilde{g}_{0}\right.\right.\right. \\
& \left.\left.\left.-Q \widetilde{G}_{\kappa}(\beta) Q\right]^{-1} Q W_{\kappa}\right) W_{\kappa}^{*} \Omega\right)
\end{aligned}
$$

follows.

For brevity, we set

$$
X^{\prime}:=W_{\kappa}^{*} Q\left[\widetilde{g}_{0}-Q G_{\kappa}(\beta) Q\right]^{-1} Q W_{\kappa}, \quad X:=W_{\kappa}^{*} Q\left[1-Q G_{\kappa}(\beta) Q\right]^{-1} Q W_{\kappa}
$$

and

$$
\tilde{X}:=W_{\kappa}^{*} Q\left[\tilde{g}_{0}-Q \widetilde{G}_{\kappa}(\beta) Q\right]^{-1} Q W_{\kappa} .
$$

Then we get

$$
\tilde{X}-X^{\prime}=-\tilde{X} X^{\prime}
$$

and hence 


$$
\tilde{X}=X^{\prime}\left(1+X^{\prime}\right)^{-1} \text { and } 1-\tilde{X}=\left(1+X^{\prime}\right)^{-1} .
$$

By definition of $W_{\kappa}$ and (1.8), one gets for large $\kappa$ the asymptotic

$$
W_{\kappa}^{*} \Omega=\sqrt{1-e^{-f}}(\pi \kappa)^{-d / 4}\left(1+O\left(\kappa^{-1}\right)\right) .
$$

By virtue of (2.18), it implies the representation

$$
g_{0}-\widetilde{g}_{0}=(\pi \kappa)^{-d / 2}\left(\sqrt{1-e^{-f}},\left(1+X^{\prime}\right)^{-1} \sqrt{1-e^{-f}}\right)\left(1+O\left(\kappa^{-1}\right)\right) .
$$

Now, we want to replace in the right-hand side of this representation the operator $X^{\prime}$ by $X$. Note that (2.11) yields $\left\|X-K_{f}\right\| \leq\left\|X-K_{f}\right\|_{1}=o(1)$. Then by $1-\widetilde{g}_{0}=O\left(\kappa^{-d / 2}\right), \widetilde{g}_{0}-g_{1}=\hat{O}\left(\kappa^{-1}\right), 1$ $-g_{1}=\hat{O}\left(\kappa^{-1}\right)$, and $\left\|W_{\kappa}^{*} \Omega\right\|=\hat{O}\left(\kappa^{-d / 4}\right)$, we find that

$$
\begin{aligned}
\left\|X^{\prime}-X\right\|= & \left(1-\widetilde{g}_{0}\right)\left\|W_{\kappa}^{*} Q\left[\widetilde{g}_{0}-Q G_{\kappa}(\beta) Q\right]^{-1}\left[1-Q G_{\kappa}(\beta) Q\right]^{-1} Q W_{\kappa}\right\| \leq \frac{1-\widetilde{g}_{0}}{\widetilde{g}_{0}-g_{1}} \| W_{\kappa}^{*} Q[1 \\
& \left.-Q G_{\kappa}(\beta) Q\right]^{-1} Q W_{\kappa}\left\|=O\left(\kappa^{1-d / 2}\right)\right\| X\left\|=O\left(\kappa^{1-d / 2}\right)\right\| K_{f} \|,
\end{aligned}
$$

which implies $\left\|X^{\prime}\right\| \leq\left\|K_{f}\right\|(1+o(1))$. Hence (2.20) yields

$$
(\pi \kappa)^{d / 2}\left(g_{0}-\widetilde{g}_{0}\right)=\left(\sqrt{1-e^{-f}},\left(1+X^{\prime}\right)^{-1} \sqrt{1-e^{-f}}\right)\left(1+O\left(\kappa^{-1}\right)\right) \geq \frac{\left\|\sqrt{1-e^{-f}}\right\|^{2}}{1+\left\|K_{f}\right\|}(1+o(1)) .
$$

Since the upper bound $(\pi \kappa)^{d / 2}\left(g_{0}-\widetilde{g}_{0}\right) \leq(\pi \kappa)^{d / 2}\left(\Omega, D_{\kappa} \Omega\right) \leq\left\|\sqrt{1-e^{-f}}\right\|^{2}$ is obvious, we get desired estimate $g_{0}-\widetilde{g}_{0}=\hat{O}\left(\kappa^{-d / 2}\right)$. Notice that the proof of the equality

$$
g_{0}-\widetilde{g}_{0}=\frac{1+o(1)}{(\pi \kappa)^{d / 2}}\left(\sqrt{1-e^{-f}},[1+X]^{-1} \sqrt{1-e^{-f}}\right)
$$

follows from (220) and the estimate,

$$
\begin{gathered}
\left|\left(\sqrt{1-e^{-f}},\left(1+X^{\prime}\right)^{-1} \sqrt{1-e^{-f}}\right)-\left(\sqrt{1-e^{-f}},(1+X)^{-1} \sqrt{1-e^{-f}}\right)\right|, \\
\leq\left\|\sqrt{1-e^{-f}}\right\|^{2}\left\|(1+X)^{-1}\right\|\left\|\left(1+X^{\prime}\right)^{-1}\right\|\left\|X-X^{\prime}\right\|=o(1) .
\end{gathered}
$$

Here $\left\|\sqrt{1-e^{-f}}\right\|$ stands for the norm in $\mathfrak{H}=L^{2}\left(\mathbb{R}^{d}\right)$, while the other $\|\cdot\|$ for the operator norm on $\mathfrak{H}$. This remark finishes the proof of the lemma.

\section{Evidence of two thermodynamic regimes}

Now we return to the conditions (2.8). We need the behavior of $r_{\kappa}$ and $\widetilde{r}_{\kappa}$ to prove the main theorem. Here we consider the behavior of $r_{\kappa}$, which classifies the phase separation. That of $\widetilde{r}_{\kappa}$ is postponed to Sec. IV.

Proposition 2.4:

(a) $\left\{r_{\kappa}\right\}$ converges to $r_{*} \in(0,1)$ for $\kappa \rightarrow \infty$, where $r_{*}$ is the unique solution of

$$
\frac{\mu}{\lambda}=\frac{\log r_{*}}{\beta \lambda}+\frac{1}{\beta^{d}} \int_{[0, \infty)^{d}} \frac{r_{*} d p}{e^{|p|_{1}-r_{*}}},
$$

if and only if $\beta^{d} \mu<\zeta(d) \lambda$.

(b) $\kappa^{d}\left(1-r_{\kappa}\right) \rightarrow \beta^{d} \lambda /\left(\beta^{d} \mu-\zeta(d) \lambda\right)$, and hence $\lim _{\kappa \rightarrow \infty} r_{\kappa}=1$, if and only if $\beta^{d} \mu>\zeta(d) \lambda$.

(c) $\lim _{\kappa \rightarrow \infty} r_{\kappa}=1$ and $\kappa^{d}\left(1-r_{\kappa}\right) \rightarrow+\infty$, if and only if $\beta^{d} \mu=\zeta(d) \lambda$.

To this end let us introduce the notation 


$$
\square_{n}^{(\kappa)}:=\frac{\beta}{\kappa}\left(n+[0,1)^{d}\right) \text { for } n \in \mathbb{Z}_{+}^{d},
$$

and define for $r \in[0,1], \nu=1,2$ and $\kappa \in[1, \infty)$, the functions $a_{\nu}(\cdot ; r), a_{\nu}^{(\kappa)}(\cdot ; r)$ on $[0, \infty)^{d}$ by

$$
a_{\nu}(p ; r):=\frac{r e^{-|p|_{1}}}{\left(1-r e^{-|p|_{1}}\right)^{\nu}}
$$

and by

$$
a_{\nu}^{(\kappa)}(p ; r):= \begin{cases}0 & \text { if } p \in \square_{0}^{(\kappa)} \\ a_{\nu}(\beta n / \kappa ; r) & \text { if } p \in \square_{n}^{(\kappa)} \text { for } n \in \mathbb{Z}_{+}^{d}-\{0\} .\end{cases}
$$

It is easy to show the following fact (see Ref. 19 for detail).

Lemma 2.5: There exists a constant $c$ which depends only on $d>2$ and $\beta>0$ such that

$$
0 \leq a_{\nu}^{(\kappa)}(p ; r) \leq a_{\nu}(c p ; 1) \in L^{1}\left([0, \infty)^{d}\right)
$$

holds for $r \in[0,1]$ and $\nu=1,2$.

Remark 2.6: If a series $\left\{r_{\kappa}\right\} \subset[0,1]$ converges to $r_{0} \in[0,1], a_{\nu}^{(\kappa)}\left(\cdot, r_{\kappa}\right) \rightarrow a_{\nu}\left(\cdot, r_{0}\right)$ holds almost everywhere (a.e.) Then the lemma and the dominated convergence theorem yield

$$
\begin{aligned}
\frac{\operatorname{Tr}\left[r_{\kappa} Q_{\kappa} G_{\kappa}(\beta) Q_{\kappa}\left(1-r_{\kappa} Q_{\kappa} G_{\kappa}(\beta) Q_{\kappa}\right)^{-\nu}\right]}{\kappa^{d}} & =\frac{1}{\kappa^{d}} \sum_{j=1}^{\infty} \frac{r_{\kappa} g_{j}^{(\kappa)}}{\left(1-r_{\kappa} g_{j}^{(\kappa)}\right)^{\nu}}=\left(\frac{1}{\beta^{d}}\right) \int_{[0, \infty)^{d}} a_{\nu}^{(\kappa)}\left(p ; r_{\kappa}\right) d p \\
& \rightarrow\left(\frac{1}{\beta^{d}}\right) \int_{[0, \infty)^{d}} a_{\nu}\left(p ; r_{0}\right) d p
\end{aligned}
$$

as $\kappa \rightarrow \infty$.

Proof of Proposition 2.4: If $r_{\kappa} \rightarrow r_{*} \in[0,1)$, then by (2.8) and the above remark one gets

$$
\begin{aligned}
\frac{\mu}{\lambda} & =\frac{\log r_{\kappa}}{\beta \lambda}+\frac{1}{\beta^{d}} \int_{[0, \infty)^{d}} a_{1}^{(\kappa)}\left(p ; r_{\kappa}\right) d p+\frac{r_{\kappa}}{\left(1-r_{\kappa}\right) \kappa^{d}} \rightarrow \frac{\log r_{*}}{\beta \lambda}+\frac{1}{\beta^{d}} \int_{[0, \infty)^{d}} a_{1}\left(p ; r_{*}\right) d p \\
& <\frac{1}{\beta^{d}} \int_{[0, \infty)^{d}} a_{1}(p ; 1) d p=\frac{\zeta(d)}{\beta^{d}} .
\end{aligned}
$$

Since equality holds at the limit actually (2.21) follows. Similarly, if $r_{\kappa} \rightarrow 1$ and $\kappa^{d}\left(1-r_{\kappa}\right) \rightarrow \infty$, then one obtains

$$
\frac{\mu}{\lambda}=\frac{\log r_{\kappa}}{\beta \lambda}+\frac{1}{\beta^{d}} \int_{[0, \infty)^{d}} a_{1}^{(\kappa)}\left(p ; r_{\kappa}\right) d p+\frac{r_{\kappa}}{\left(1-r_{\kappa}\right) \kappa^{d}} \rightarrow \frac{1}{\beta^{d}} \int_{[0, \infty)^{d}} a_{1}(p ; 1)=\frac{\zeta(d)}{\beta^{d}} .
$$

Finally, if $\kappa^{d}\left(1-r_{\kappa}\right) \rightarrow \alpha>0$, we get

$$
\frac{\mu}{\lambda}=\frac{1}{\alpha}+\frac{\zeta(d)}{\beta^{d}}>\frac{\zeta(d)}{\beta^{d}} \quad \text { and } \quad \alpha=\frac{\beta^{d} \lambda}{\beta^{d} \mu-\zeta(d) \lambda} .
$$

On the other hand, if $\left\{r_{k}\right\}$ does not converge, by compactness we can take two convergent subsequences $\left\{r_{\kappa_{i}}\right\}(i=1,2)$ with different limits $0<r^{(1)}<r^{(2)}<1$. Then above arguments yield

$$
\frac{\log r^{(1)}}{\beta \lambda}+\frac{1}{\beta^{d}} \int_{[0, \infty)^{d}} a_{1}\left(p ; r^{(1)}\right) d p=\frac{\log r^{(2)}}{\beta \lambda}+\frac{1}{\beta^{d}} \int_{[0, \infty)^{d}} a_{1}\left(p ; r^{(2)}\right) d p,
$$

which contradicts to the strict monotonicity of the function 


$$
h(r)=\frac{\log r}{\beta \lambda}+\frac{1}{\beta^{d}} \int_{[0, \infty)^{d}} a_{1}(p ; r) d p .
$$

Similar arguments are valid for the cases $r^{(1)}=0$ or $r^{(2)}=1$. If $r_{\kappa} \rightarrow 1$ but $\kappa^{d}\left(1-r_{\kappa}\right)$ does not converge, we again get a contradiction.

\section{PROOF OF THEOREM 1.2}

\section{A. The case $\mu \leq \mu_{c}(\beta)$ (normal phase)}

Let us recall that for the weak convergence of random point fields, it is enough to prove the convergence of the generating functionals. Therefore, we have to evaluate the integral in (2.4). We begin with estimates of the Fredholm determinant in the integrand. For all values of $x$, we have

$$
\begin{aligned}
\left|\operatorname{det}\left(1-\left(e^{i x}-1\right) \frac{r_{\kappa} G_{\kappa}(\beta)}{1-r_{\kappa} G_{\kappa}(\beta)}\right)\right|^{2} & =\operatorname{det}\left[\left(1-\left(e^{i x}-1\right) \frac{r_{\kappa} G_{\kappa}(\beta)}{1-r_{\kappa} G_{\kappa}(\beta)}\right)\left(1-\left(e^{-i x}-1\right) \frac{r_{\kappa} G_{\kappa}(\beta)}{1-r_{\kappa} G_{\kappa}(\beta)}\right)\right] \\
& =\operatorname{det}\left[1+4 \sin ^{2} \frac{x}{2} \frac{r_{\kappa} G_{\kappa}(\beta)}{\left(1-r_{\kappa} G_{\kappa}(\beta)\right)^{2}}\right] \geq 1
\end{aligned}
$$

Set $\alpha \in(d / 3, d / 2)$. Then for $|x| \leq \kappa^{-\alpha}$, we have

$$
\operatorname{det}\left[1-\left(e^{i x}-1\right) \frac{r_{\kappa} G_{\kappa}(\beta)}{1-r_{\kappa} G_{\kappa}(\beta)}\right]^{-1}=\exp \left[i x \operatorname{Tr} \frac{r_{\kappa} G_{\kappa}(\beta)}{1-r_{\kappa} G_{\kappa}(\beta)}-\frac{x^{2}}{2} \operatorname{Tr} \frac{r_{\kappa} G_{\kappa}(\beta)}{\left(1-r_{\kappa} G_{\kappa}(\beta)\right)^{2}}\right]\left(1+O\left(\kappa^{d-3 \alpha}\right)\right),
$$

In fact,

$$
\begin{aligned}
\log \text { of the left hand side }= & -\sum_{n=0}^{\infty} \log \left(1-\left(e^{i x}-1\right) \frac{r_{\kappa} g_{n}^{(\kappa)}}{1-r_{\kappa} g_{n}^{(\kappa)}}\right)=\left(e^{i x}-1\right) \operatorname{Tr} \frac{r_{\kappa} G_{\kappa}(\beta)}{1-r_{\kappa} G_{\kappa}(\beta)} \\
& +\frac{\left(e^{i x}-1\right)^{2}}{2} \operatorname{Tr}\left(\frac{r_{\kappa} G_{\kappa}(\beta)}{1-r_{\kappa} G_{\kappa}(\beta)}\right)^{2}+R_{1},
\end{aligned}
$$

where $R_{1}=O\left(\kappa^{d-3 \alpha}\right)$ since $r_{\kappa} \rightarrow r_{*} \in(0,1)$ and

$$
\operatorname{Tr}\left[\frac{r_{\kappa} G_{\kappa}(\beta)}{1-r_{\kappa} G_{\kappa}(\beta)}\right]^{\ell} \leq\left(\frac{r_{\kappa} g_{0}^{(\kappa)}}{1-r_{\kappa} g_{0}^{(\kappa)}}\right)^{\ell-1} \operatorname{Tr} \frac{r_{\kappa} G_{\kappa}(\beta)}{1-r_{\kappa} G_{\kappa}(\beta)}=O\left(\kappa^{d}\right) .
$$

For the last equality, we recall (2.8) and Proposition 2.4(a).

Similarly, we have

$$
\left(e^{i x}-1\right) \operatorname{Tr} \frac{r_{\kappa} G_{\kappa}(\beta)}{1-r_{\kappa} G_{\kappa}(\beta)}=\left(i x-\frac{x^{2}}{2}\right) \operatorname{Tr} \frac{r_{\kappa} G_{\kappa}(\beta)}{1-r_{\kappa} G_{\kappa}(\beta)}+O\left(\kappa^{d-3 \alpha}\right)
$$

and

$$
\frac{\left(e^{i x}-1\right)^{2}}{2} \operatorname{Tr}\left(\frac{r_{\kappa} G_{\kappa}(\beta)}{1-r_{\kappa} G_{\kappa}(\beta)}\right)^{2}=-\frac{x^{2}}{2} \operatorname{Tr}\left(\frac{r_{\kappa} G_{\kappa}(\beta)}{1-r_{\kappa} G_{\kappa}(\beta)}\right)^{2}+O\left(\kappa^{d-3 \alpha}\right) .
$$

Thus we get (3.2) and the following lemma.

Lemma 3.1.

$$
\Xi_{\kappa}(\beta, \mu)=\frac{e^{\kappa^{d}\left(\beta \mu-\log r_{\kappa}\right)^{2} / 2 \beta \lambda}\left(1+O\left(\kappa^{d-3 \alpha}\right)\right)}{\sqrt{1+\beta \lambda \kappa^{-d} \operatorname{Tr}\left[r_{\kappa} G_{\kappa}(\beta)\left(1-r_{\kappa} G_{\kappa}(\beta)\right)^{-2}\right]} \operatorname{Det}\left(1-r_{\kappa} G_{\kappa}(\beta)\right)} .
$$

Proof: From the above estimates and (2.3), we have 


$$
\begin{array}{rl}
\int_{\mathbb{R}} & d x \frac{e^{-i s_{\kappa} x-\kappa^{d} x^{2} / 2 \beta \lambda}}{\operatorname{Det}\left[1-\left(e^{i x}-1\right) r_{\kappa} G_{\kappa}(\beta)\left(1-r_{\kappa} G_{\kappa}(\beta)\right)^{-1}\right]} \\
& =\int_{-\kappa^{-\alpha}}^{\kappa^{-\alpha}} d x e^{-\kappa^{d} x^{2} / 2 \beta \lambda-i s_{\kappa} x+i x \operatorname{Tr}\left[r_{\kappa} G_{\kappa}(\beta)\left(1-r_{\kappa} G_{\kappa}(\beta)\right)^{-1}\right]-x^{2} \operatorname{Tr}\left[r_{\kappa} G_{\kappa}(\beta)\left(1-r_{\kappa} G_{\kappa}(\beta)\right)^{-2}\right] / 2}\left(1+O\left(\kappa^{d-3 \alpha}\right)\right)+R_{2} \\
& =\int_{-\infty}^{\infty} d x \exp \left[-\left(\frac{\kappa^{d}}{\beta \lambda}+\operatorname{Tr} \frac{r_{\kappa} G_{\kappa}(\beta)}{\left(1-r_{\kappa} G_{\kappa}(\beta)\right)^{2}}\right) \frac{x^{2}}{2}\right]\left(1+O\left(\kappa^{d-3 \alpha}\right)\right)+R_{2}+R_{3},
\end{array}
$$

where

$$
R_{2}=\int_{|x|>\kappa^{-\alpha}} \frac{e^{-i s_{\kappa} x-\kappa^{d} x^{2} / 2 \beta \lambda}}{\operatorname{det}\left[1-\left(e^{i x}-1\right) r_{\kappa} G_{\kappa}(\beta)\left(1-r_{\kappa} G_{\kappa}(\beta)\right)^{-1}\right]}=O\left(e^{-\widetilde{c} \kappa^{d-2 \alpha}}\right)
$$

for some $\tilde{c}>0$, thanks to $(3.1)$, and

$$
R_{3}=-\int_{|x|>\kappa^{-\alpha}} d x \exp \left[-\left(\frac{\kappa^{d}}{\beta \lambda}+\operatorname{Tr} \frac{r_{\kappa} G_{\kappa}(\beta)}{\left(1-r_{\kappa} G_{\kappa}(\beta)\right)^{2}}\right) \frac{x^{2}}{2}\right]\left(1+O\left(\kappa^{d-3 \alpha}\right)\right)=O\left(e^{-\tilde{c}^{\prime} \kappa^{d-2 \alpha}}\right)
$$

for some $\widetilde{c}^{\prime}>0$. Then, the lemma follows from (2.4) and (2.3).

For $\widetilde{\Xi}_{\kappa}(\beta, \mu)$, we have the following asymptotics:

Lemma 3.2.

$$
\widetilde{\Xi}_{\kappa}(\beta, \mu)=\frac{e^{\kappa^{d}\left(\beta \mu-\log \widetilde{r}_{\kappa}\right)^{2} / 2 \beta \lambda}\left(1+O\left(\kappa^{d-3 \alpha}\right)\right)}{\sqrt{1+\beta \lambda \kappa^{-d} \operatorname{Tr}\left[\widetilde{r}_{\kappa} \widetilde{G}_{\kappa}(\beta)\left(1-\widetilde{r}_{\kappa} \widetilde{G}_{\kappa}(\beta)\right)^{-2}\right] \operatorname{Det}\left(1-\widetilde{r}_{\kappa} \widetilde{G}_{\kappa}(\beta)\right)}} .
$$

To show the formula, we note that "tilded" quantities are close to corresponding "untilded" ones. In fact, the following asymptotics is established. Then it is obvious to get Lemma 3.2 by a similar argument.

Lemma 3.3: For large $\kappa$ one gets

(i)

$$
\tilde{r}_{\kappa} \geq r_{\kappa}
$$

(ii)

$$
\widetilde{r}_{\kappa}-r_{\kappa}=O\left(\kappa^{-d}\right),
$$

(iii)

$$
\operatorname{Tr}\left[\frac{r_{\kappa} G_{\kappa}(\beta)}{1-r_{\kappa} G_{\kappa}(\beta)}\right]=\hat{O}\left(\kappa^{d}\right), \quad \operatorname{Tr}\left[\frac{\widetilde{r}_{\kappa} \widetilde{G}_{\kappa}(\beta)}{1-\widetilde{r}_{\kappa} \widetilde{G}_{\kappa}(\beta)}\right]=\hat{O}\left(\kappa^{d}\right),
$$

and

(iv)

$$
\operatorname{Tr}\left[\frac{r_{\kappa} G_{\kappa}(\beta)}{\left(1-r_{\kappa} G_{\kappa}(\beta)\right)^{2}}\right]-\operatorname{Tr}\left[\frac{\widetilde{r}_{\kappa} \widetilde{G}_{\kappa}(\beta)}{\left(1-\widetilde{r}_{\kappa} \widetilde{G}_{\kappa}(\beta)\right)^{2}}\right]=O(1)
$$

Proof:

(i) Let $h_{\kappa}$ be the functions on $(0,1)$ defined by 


$$
h_{\kappa}(r)=\frac{\log r}{\beta \lambda}+\frac{1}{\kappa^{d}} \operatorname{Tr} \frac{r G_{\kappa}(\beta)}{1-r G_{\kappa}(\beta)}=\frac{\log r}{\beta \lambda}+\frac{r}{\kappa^{d}(1-r)}+\frac{1}{\beta^{d}} \int_{[0, \infty)^{d}} a_{1}^{(\kappa)}(p ; r) d p .
$$

We also introduce the function $\widetilde{h}_{\kappa}$ on $\left(0, \widetilde{g}_{0}^{(\kappa)-1}\right)$ by

$$
\tilde{h}_{\kappa}(r)=\frac{\log r}{\beta \lambda}+\frac{1}{\kappa^{d}} \operatorname{Tr} \frac{r \widetilde{G}_{\kappa}(\beta)}{1-r \widetilde{G}_{\kappa}(\beta)} .
$$

Since $G_{\kappa}(\beta) \geq \widetilde{G}_{\kappa}(\beta), h_{\kappa} \geq \widetilde{h}_{\kappa}$ follows. Obviously $h_{\kappa}$ and $\widetilde{h}_{\kappa}$ are strictly increasing continuous functions. Then we have $r_{\kappa} \leq \widetilde{r}_{\kappa}$ because $r_{\kappa}$ and $\widetilde{r}_{\kappa}$ are solutions of $h_{\kappa}(r)=\mu / \lambda$ and $\tilde{h}_{\kappa}(r)$ $=\mu / \lambda$, respectively. [Recall (2.8) and (2.9).]

(ii) From $\widetilde{h}_{\kappa}\left(\widetilde{r}_{\kappa}\right)=h_{\kappa}\left(r_{\kappa}\right)$, we have

$$
\begin{aligned}
\frac{1}{\beta \lambda} \log \frac{\widetilde{r}_{\kappa}}{r_{\kappa}} \leq & \widetilde{h}_{\kappa}\left(\widetilde{r}_{\kappa}\right)-\widetilde{h}_{\kappa}\left(r_{\kappa}\right)=h_{\kappa}\left(r_{\kappa}\right)-\widetilde{h}_{\kappa}\left(r_{\kappa}\right)=\frac{1}{\kappa^{d}} \operatorname{Tr}\left[\frac { 1 } { 1 - r _ { \kappa } \widetilde { G } _ { \kappa } ( \beta ) } \left(G_{\kappa}(\beta)\right.\right. \\
& \left.\left.-\widetilde{G}_{\kappa}(\beta)\right) \frac{r_{\kappa}}{1-r_{\kappa} G_{\kappa}(\beta)}\right]=O\left(\kappa^{-d}\right) .
\end{aligned}
$$

Here we have used Lemma 2.3 and the fact that $r_{\kappa}$ is bounded away from 1 . The desired estimate follows.

(iii) Since we already know that $r_{\kappa}, \widetilde{r}_{\kappa} \rightarrow r_{*} \in(0,1)$, we get these estimates readily from (2.8) and (2.9).

(iv) This can be derived by the telescoping together with (ii) and Lemma 2.3.

Now, let us consider the limit of the ratio $\widetilde{\Xi}_{\kappa}(\beta, \mu) / \Xi_{\kappa}(\beta, \mu)$ to derive (1.27). From Lemmas 3.1 and 3.2 , we have

$$
\begin{aligned}
E_{\beta, r_{*}}\left[e^{-\langle f, \xi\rangle}\right]= & \lim _{\kappa \rightarrow \infty} \frac{\widetilde{\Xi}_{\kappa}(\beta, \mu)}{\Xi_{\kappa}(\beta, \mu)}=\lim _{\kappa \rightarrow \infty} \sqrt{\frac{1+\beta \lambda \kappa^{-d} \operatorname{Tr}\left[r_{\kappa} G_{\kappa}(\beta)\left(1-r_{\kappa} G_{\kappa}(\beta)\right)^{-2}\right]}{1+\beta \lambda \kappa^{-d} \operatorname{Tr}\left[\widetilde{r}_{\kappa} \widetilde{G}_{\kappa}(\beta)\left(1-\widetilde{r}_{\kappa} \widetilde{G}_{\kappa}(\beta)\right)^{-2}\right]}} \\
& \times \frac{\operatorname{det}\left[1-\widetilde{r}_{\kappa} G_{\kappa}(\beta)\right]}{\operatorname{det}\left[1-\widetilde{r}_{\kappa} \widetilde{G}_{\kappa}(\beta)\right]} \frac{\operatorname{det}\left[1-r_{\kappa} G_{\kappa}(\beta)\right]}{\operatorname{det}\left[1-\widetilde{r}_{\kappa} G_{\kappa}(\beta)\right]} e^{\kappa^{d}(2 \beta \lambda)^{-1}\left[\left(\beta \mu-\log \widetilde{r}_{\kappa}\right)^{2}-\left(\beta \mu-\log r_{\kappa}\right)^{2}\right]} .
\end{aligned}
$$

Lemma 3.3 yields that the first factor is equal to $1+O\left(\kappa^{-d}\right)$. For the second factor, we note that

$$
\begin{aligned}
& \left\|\sqrt{1-e^{-f}} \frac{\widetilde{r}_{\kappa} G_{\kappa}(\beta)}{1-\widetilde{r}_{\kappa} G_{\kappa}(\beta)} \sqrt{1-e^{-f}}-\sqrt{1-e^{-f}} \frac{r_{*} G_{\kappa}(\beta)}{1-r_{*} G_{\kappa}(\beta)} \sqrt{1-e^{-f}}\right\|_{1} \\
& =\left\|\sqrt{1-e^{-f}} \sqrt{\frac{r_{*} G_{\kappa}(\beta)}{1-r_{*} G_{\kappa}(\beta)}} \frac{r_{*}-\widetilde{r}_{\kappa}}{r_{*}} \frac{1}{1-\widetilde{r}_{\kappa} G_{\kappa}(\beta)} \sqrt{\frac{r_{*} G_{\kappa}(\beta)}{1-r_{*} G_{\kappa}(\beta)}} \sqrt{1-e^{-f}}\right\|_{1} \\
& \leq \frac{r_{*}-\widetilde{r}_{\kappa}}{r_{*}\left(1-\widetilde{r}_{\kappa}\right)}\left\|\sqrt{1-e^{-f}} \frac{r_{*} G_{\kappa}(\beta)}{1-r_{*} G_{\kappa}(\beta)} \sqrt{1-e^{-f}}\right\|_{1}^{\rightarrow} \rightarrow 0
\end{aligned}
$$

holds, where $\|\cdot\|_{1}$ stands for the trace norm. Together with (2.10), 


$$
\begin{aligned}
\frac{\operatorname{det}\left[1-\tilde{r}_{\kappa} \widetilde{G}_{\kappa}(\beta)\right]}{\operatorname{det}\left[1-\widetilde{r}_{\kappa} G_{\kappa}(\beta)\right]} & =\operatorname{det}\left[1+\widetilde{r}_{\kappa}\left(G_{\kappa}(\beta)-\widetilde{G}_{\kappa}(\beta)\right)\left(1-\tilde{r}_{\kappa} G_{\kappa}(\beta)\right)^{-1}\right] \\
& =\operatorname{det}\left[1+\sqrt{1-e^{-f}} \frac{\tilde{r}_{\kappa} G_{\kappa}(\beta)}{1-\tilde{r}_{\kappa} G_{\kappa}(\beta)} \sqrt{1-e^{-f}}\right] \\
& \rightarrow \operatorname{det}\left[1+\sqrt{1-e^{-f}} \frac{r_{*} G(\beta)}{1-r_{*} G(\beta)} \sqrt{1-e^{-f}}\right]
\end{aligned}
$$

follows. Similar to the third factor, we have

$$
\begin{aligned}
\frac{\operatorname{det}\left[1-\tilde{r}_{\kappa} G_{\kappa}(\beta)\right]}{\operatorname{det}\left[1-r_{\kappa} G_{\kappa}(\beta)\right]} & =\operatorname{det}\left[1-\frac{\tilde{r}_{\kappa}-r_{\kappa}}{r_{\kappa}} \frac{r_{\kappa} G_{\kappa}(\beta)}{1-r_{\kappa} G_{\kappa}(\beta)}\right]=\exp \left(-\frac{\tilde{r}_{\kappa}-r_{\kappa}}{r_{\kappa}} \operatorname{Tr}\left[\frac{r_{\kappa} G_{\kappa}(\beta)}{1-r_{\kappa} G_{\kappa}(\beta)}\right]+O\left(\kappa^{-d}\right)\right) \\
& =\exp \left(-\frac{\tilde{r}_{\kappa}-r_{\kappa}}{r_{\kappa}} \frac{\kappa^{d}}{\beta \lambda}\left(\beta \mu-\log r_{\kappa}\right)+O\left(\kappa^{-d}\right)\right),
\end{aligned}
$$

where we have used Lemma 3.3 and (2.8). It also follows from Lemma 3.3 that the fourth factor is equal to

$$
\exp \left[\frac{\kappa^{d}}{2 \beta \lambda}\left(2 \beta \mu-\log r_{\kappa} \tilde{r}_{\kappa}\right) \log \left(1+\frac{r_{\kappa}-\tilde{r}_{\kappa}}{\tilde{r}_{\kappa}}\right)\right]=\exp \left[-\frac{\tilde{r}_{\kappa}-r_{\kappa}}{r_{\kappa}} \frac{\kappa^{d}}{\beta \lambda}\left(\beta \mu-\log r_{\kappa}\right)+O\left(\kappa^{-d}\right)\right] .
$$

Thus we get (1.27). Since convergence of generating functionals yields the weak convergence of random point fields, Theorem 1.2(i) follows.

\section{B. The case $\mu>\mu_{c}(\beta)$ (condensed phase)}

Let us set

$$
p_{j}^{(\kappa)}=\frac{r_{\kappa} g_{j}^{(\kappa)}}{1-r_{\kappa} g_{j}^{(\kappa)}}, \quad \varepsilon_{j}^{(\kappa)}=\log \left(1+\frac{1}{p_{j}^{(\kappa)}}\right) \quad \text { and } \quad \delta^{(\kappa)}=\log \left(1+\frac{1}{2 p_{1}^{(\kappa)}}\right)
$$

for $j=0,1, \ldots$. Then it follows from (2.3) and Proposition 2.4(b) that

$$
s_{\kappa}=\sum_{j=0}^{\infty} p_{j}^{(\kappa)}, \quad 1+p_{0}^{(\kappa)}=\frac{1}{1-r_{\kappa}}=\frac{\kappa^{d}\left(\beta^{d} \mu-\zeta(d) \lambda\right)}{\beta^{d} \lambda}(1+o(1))=\hat{O}\left(\kappa^{d}\right), \quad \varepsilon_{0}^{(\kappa)}=\hat{O}\left(\kappa^{-d}\right) .
$$

Since $g_{1}^{(\kappa)}=e^{-\beta / \kappa}$, we have

$$
p_{1}^{(\kappa)}=\hat{O}(\kappa), \quad \delta^{(\kappa)}=\hat{O}\left(\kappa^{-1}\right), \quad \varepsilon_{1}^{(\kappa)}=\hat{O}\left(\kappa^{-1}\right)
$$

and

$$
\varepsilon_{0}^{(\kappa)}<\delta^{(\kappa)}<\varepsilon_{1}^{(\kappa)} \leq \varepsilon_{2}^{(\kappa)} \leq \cdots
$$

Note also that $s_{\kappa}=\hat{O}\left(\kappa^{d}\right)$ holds because of (2.3) and (2.8). By Remark 2.6 and $r_{\kappa} g_{j}^{\kappa} \in(0,1)$

$$
\sum_{j=1}^{\infty} p_{j}^{(\kappa) 2}=O\left(\kappa^{d}\right)
$$

and (A10), we get

$$
\prod_{j=1}^{\infty}\left(1-\frac{p_{j}^{(\kappa)}}{p_{0}^{(\kappa)}}\right)=\exp \left(\sum_{j=1}^{\infty} \log \left(1-\frac{p_{j}^{(\kappa)}}{p_{0}^{(\kappa)}}\right)\right)=\exp \left(-\frac{s_{\kappa}-p_{0}^{(\kappa)}}{p_{0}^{(\kappa)}}+O\left(\kappa^{-d}\right)\right) .
$$

Similarly, we have 


$$
\prod_{j=1}^{\infty}\left(1-p_{j}^{(\kappa)}\left(e^{\delta^{(\kappa)}}-1\right)\right)=\exp \left(-\sum_{j=1}^{\infty} p_{j}^{(\kappa)}\left(e^{\delta^{(\kappa)}}-1\right)+O\left(\kappa^{d-2}\right)\right)=\exp \left(-\delta^{(\kappa)}\left(s_{\kappa}-p_{0}^{(\kappa)}\right)+O\left(\kappa^{d-2}\right)\right)
$$

since $p_{j}^{(\kappa)}\left(e^{\delta^{(\kappa)}}-1\right) \leq p_{1}^{(\kappa)}\left(e^{\delta^{(\kappa)}}-1\right)=1 / 2$ for $j=1,2, \ldots$

Lemma 3.4: For large $\kappa$ we have the asymptotics

$$
\Xi_{\kappa}(\beta, \mu)=\sqrt{\frac{2 \pi \beta \lambda}{e^{2} \kappa^{d}}} \frac{\beta^{d-1} e^{\kappa^{d}\left(\beta \mu-\log r_{\kappa}\right)^{2} / 2 \beta \lambda}}{\left(\beta^{d} \mu-\zeta(d) \lambda\right) \operatorname{Det}\left(1-r_{\kappa} G_{\kappa}(\beta)\right)}(1+o(1)) .
$$

Proof: As in the proof of Lemma 3.1, we start with the integral

$$
\begin{aligned}
\mathcal{I} & =\int_{\mathrm{R}} d x \frac{e^{-i s_{\kappa} x-\kappa^{d} x^{2} / 2 \beta \lambda}}{\operatorname{det}\left[1-\left(e^{i x}-1\right) r_{\kappa} G_{\kappa}(\beta)\left(1-r_{\kappa} G_{\kappa}(\beta)\right)^{-1}\right]} \\
& =\int_{-\infty}^{\infty} d z \frac{e^{-i s_{\kappa} z-\kappa^{d} z^{2} / 2 \beta \lambda}}{\prod_{j=0}^{\infty}\left(1-\left(e^{i z}-1\right) p_{j}^{(\kappa)}\right)}=\left(\int_{-\infty}^{\infty}-\int_{-\infty-i \delta^{(\kappa)}}^{\infty-i \delta^{(\kappa)}}\right)+\int_{-\infty-i \delta^{(\kappa)}}^{\infty-i \delta^{(\kappa)}}=\mathcal{I}_{1}+\mathcal{I}_{2} .
\end{aligned}
$$

Since $1+p_{0}^{(\kappa)}-e^{i z} p_{0}^{(\kappa)}=\left(1+p_{0}^{(\kappa)}\right)\left(1-e^{i\left(z-2 n \pi+i \epsilon_{0}^{(\kappa)}\right)}\right)$, the integrand of $\mathcal{I}_{1}$ has simple poles at $z=2 n \pi$ $-i \epsilon_{0}^{(\kappa)}(n \in \mathbb{Z})$. Let us calculate $\mathcal{I}_{1}$ by means of residues

$$
\begin{aligned}
\mathcal{I}_{1} & =-2 \pi i \sum_{n=-\infty}^{\infty} \operatorname{res}\left[\frac{1}{\left(1+p_{0}^{(\kappa)}\right)\left(1-e^{i\left(z-2 n \pi+i \epsilon_{0}^{(\kappa)}\right)}\right)} \frac{e^{-i s_{\kappa^{z}}-\kappa^{d} z^{2} / 2 \beta \lambda}}{\prod_{j=1}^{\infty}\left(1-\left(e^{i z}-1\right) p_{j}^{(\kappa)}\right)} ; 2 n \pi-i \epsilon_{0}^{(\kappa)}\right] \\
& =\frac{2 \pi e^{-S_{\kappa} \epsilon_{0}^{(\kappa)}+\kappa^{d} \epsilon_{0}^{(\kappa) 2} / 2 \beta \lambda}}{\left(1+p_{0}^{(\kappa)}\right) \prod_{j=1}^{\infty}\left(1-p_{j}^{(\kappa)} / p_{0}^{(\kappa)}\right)}\left(1+O\left(e^{-2 \pi^{2} \kappa^{d} / \beta \lambda}\right)\right)=\frac{2 \pi \beta^{d} \lambda e^{-1+O\left(\kappa^{-d}\right)}}{\kappa^{d}\left(\beta^{d} \mu-\zeta(d) \lambda\right)} .
\end{aligned}
$$

Here the pole $z=-i \epsilon_{0}^{(\kappa)}$ gives the dominant contribution in the second equality. In the third equality, we have used the relations above this lemma.

On the other hand, we have

$$
\left|\mathcal{I}_{2}\right| \leq \int_{-\infty}^{\infty} d x \frac{e^{-s_{\kappa} \delta^{(\kappa)}-\kappa^{d}\left(x^{2}-\delta^{(\kappa) 2}\right) / 2 \beta \lambda}}{\prod_{j=0}^{\infty}\left|\left(1-\left(e^{i x+\delta^{(\kappa)}}-1\right) p_{j}^{(\kappa)}\right)\right|} \leq \sqrt{\frac{2 \pi \beta \lambda}{\kappa^{d}}} \frac{e^{-\hat{O}\left(\kappa^{d-1}\right)}}{\left(e^{\delta^{(\kappa)}}-1\right) p_{0}^{(\kappa)}-1}=o\left(\mathcal{I}_{1}\right),
$$

where we have used

$$
\begin{aligned}
\prod_{j=0}^{\infty}\left|\left(1-\left(e^{i x+\delta^{(\kappa)}}-1\right) p_{j}^{(\kappa)}\right)\right| & \geq\left(\left(e^{\delta^{(\kappa)}}-1\right) p_{0}^{(\kappa)}-1\right) \prod_{j=1}^{\infty}\left|\left(1-\left(e^{\delta^{(\kappa)}}-1\right) p_{j}^{(\kappa)}\right)\right| \\
& \geq\left(\left(e^{\delta^{(\kappa)}}-1\right) p_{0}^{(\kappa)}-1\right) \exp \left(-\delta^{(\kappa)}\left(s_{\kappa}-p_{0}^{(\kappa)}\right)+O\left(\kappa^{d-2}\right)\right),
\end{aligned}
$$

which follows from

$$
1-\left(e^{i x+\delta^{(\kappa)}}-1\right) p_{j}^{(\kappa)}=\left(1+p_{j}^{(\kappa)}\right)\left(1-e^{i x+\delta^{(\kappa)}-\varepsilon_{j}^{(\kappa)}}\right)
$$

and $\delta^{(\kappa)}-\varepsilon_{0}^{(\kappa)}>0, \delta^{(\kappa)}-\varepsilon_{j}^{(\kappa)}<0$, and $(j=1,2, \ldots)$. Note also that $\delta^{(\kappa)} p_{0}^{(\kappa)}=\hat{O}\left(\kappa^{d-1}\right)$ holds. From (2.4) and the first equality in (2.3), one gets desired expression for the asymptotics of $\Xi_{\kappa}(\beta, \mu)$.

In order to obtain the corresponding asymptotics for $\widetilde{\Xi}_{\kappa}(\beta, \mu)$, we use the following estimates of $\widetilde{p}_{j}^{(\kappa)}=\widetilde{r}_{\kappa} \widetilde{g}_{j}^{(\kappa)} /\left(1-\widetilde{r}_{\kappa} \widetilde{g}_{j}^{(\kappa)}\right)(j=0,1, \ldots)$.

Lemma 3.5:

$$
1-\widetilde{r}_{\kappa} \widetilde{g}_{0}^{(\kappa)}=\frac{\beta^{d} \lambda(1+o(1))}{\kappa^{d}\left(\beta^{d} \mu-\zeta(d) \lambda\right)}, \quad\left|1-\widetilde{r}_{\kappa}\right|=O\left(\kappa^{-d / 2}\right),
$$




$$
\widetilde{p}_{0}^{(\kappa)}=\hat{O}\left(\kappa^{d}\right), \quad \widetilde{p}_{1}^{(\kappa)}=\hat{O}(\kappa), \quad \sum_{j=1}^{\infty} \widetilde{p}_{j}^{(\kappa)}=\hat{O}\left(\kappa^{d}\right), \quad \sum_{j=1}^{\infty} \widetilde{p}_{j}^{(\kappa) 2}=O\left(\kappa^{d}\right) .
$$

Proof: Proposition 2.4(b), Lemma 3.3(i), and Lemma 2.3 yield

$$
1-\hat{O}\left(\kappa^{-d}\right)=r_{\kappa} \leq \widetilde{r}_{\kappa}<\widetilde{g}_{0}^{(\kappa)-1}=1+\hat{O}\left(\kappa^{-d / 2}\right),
$$

which implies $\left|1-\widetilde{r}_{\kappa}\right|=O\left(\kappa^{-d / 2}\right)$. Note that the argument which shows $r_{\kappa} \leq \widetilde{r}_{\kappa}$ in the proof of Lemma 3.3 is also valid for the present case. In the variational formula

$$
\widetilde{g}_{1}^{(\kappa)}=\sup _{\psi \perp \widetilde{\Omega}} \frac{\left(\psi, \widetilde{G}_{\kappa}(\beta) \psi\right)}{(\psi, \psi)}=\sup _{\psi \perp \tilde{\Omega}} \frac{\left[\left(\psi, G_{\kappa}(\beta) \psi\right)-\left(\psi, D_{\kappa} \psi\right)\right]}{(\psi, \psi)},
$$

we can use as $\psi$ a linear combination of two eigenfunctions $\kappa^{-d / 4} \phi_{s}(x / \sqrt{\kappa})$ of $G_{\kappa}(\beta)$ perpendicular to $\widetilde{\Omega}$ [e.g., with $s=(1,0,0, \ldots)$ and $s=(0,1,0, \ldots)]$. Then we get $\widetilde{g}_{1}^{\kappa} \geq 1-\hat{O}\left(\kappa^{-1}\right)$. Together with $\widetilde{g}_{1}^{(\kappa)} \leq g_{1}^{(\kappa)}=1-\hat{O}\left(\kappa^{-1}\right), \widetilde{g}_{1}^{(\kappa)}=1-\hat{O}\left(\kappa^{-1}\right)$ follows. Thus $\hat{O}(\kappa)=\widetilde{p}_{1}^{(\kappa)} \geq \widetilde{p}_{2}^{(\kappa)} \geq \cdots$ holds.

Now we get

$$
\left|\sum_{j=1}^{\infty} \widetilde{p}_{j}^{(\kappa)}-\sum_{j=1}^{\infty} \widetilde{g}_{j}^{(\kappa)} /\left(1-\widetilde{g}_{j}^{(\kappa)}\right)\right|=\left|1-\widetilde{r}_{\kappa}\right| \sum_{j=1}^{\infty} \widetilde{g}_{j}^{(\kappa)} /\left(\left(1-\widetilde{r}_{\kappa} \widetilde{g}_{j}^{(\kappa)}\right)\left(1-\widetilde{g}_{j}^{(\kappa)}\right)\right)=O\left(\kappa^{1-d / 2}\right) \sum_{j=1}^{\infty} \widetilde{g}_{j}^{(\kappa)} /\left(1-\widetilde{g}_{j}^{(\kappa)}\right),
$$

which implies

$$
\sum_{j=1}^{\infty} \widetilde{p}_{j}^{(\kappa)}=\left(1+O\left(\kappa^{1-d / 2}\right)\right) \sum_{j=1}^{\infty} \widetilde{g}_{j}^{(\kappa)} /\left(1-\widetilde{g}_{j}^{(\kappa)}\right) .
$$

On the other hand, because

$$
\left|\sum_{j=1}^{\infty} \frac{\widetilde{g}_{j}^{(\kappa)}}{1-\widetilde{g}_{j}^{(\kappa)}}-\sum_{j=1}^{\infty} \frac{g_{j}^{(\kappa)}}{1-g_{j}^{(\kappa)}}\right| \leq \sum_{j=1}^{\infty} \frac{g_{j}^{(\kappa)}-\widetilde{g}_{j}^{(\kappa)}}{\left(1-g_{1}^{(\kappa)}\right)\left(1-\widetilde{g}_{1}^{(\kappa)}\right)} \leq \frac{\operatorname{Tr} D_{\kappa}}{\left(1-g_{1}^{(\kappa)}\right)\left(1-\widetilde{g}_{1}^{(\kappa)}\right)}=O\left(\kappa^{2}\right),
$$

we have

$$
\frac{1}{\kappa^{d}} \sum_{j=1}^{\infty} \frac{\widetilde{g}_{j}^{(\kappa)}}{1-\widetilde{g}_{j}^{(\kappa)}}=\frac{1}{\kappa^{d}} \sum_{j=1}^{\infty} \frac{g_{j}^{(\kappa)}}{1-g_{j}^{(\kappa)}}+O\left(\kappa^{2-d}\right)=\frac{\zeta(d)}{\beta^{d}}+o(1)
$$

where we recall Remark 2.6. Thus we have $\kappa^{-d} \sum_{j=1}^{\infty} \widetilde{p}_{j}^{(\kappa)}=\beta^{-d} \zeta(d)+o(1)$. Using (2.9), we get

$$
\frac{\widetilde{r}_{\kappa} \widetilde{g}_{0}^{(\kappa)}}{\kappa^{d}\left(1-\widetilde{r}_{\kappa} \widetilde{g}_{0}^{(\kappa)}\right)}=-\frac{\log \widetilde{r}_{\kappa}}{\beta \lambda}+\frac{\mu}{\lambda}-\frac{1}{\kappa^{d}} \sum_{j=1}^{\infty} \widetilde{p}_{j}^{(\kappa)}=\frac{\mu}{\lambda}-\frac{\zeta(d)}{\beta^{d}}+o(1),
$$

which yields the first and the third equality.

To prove the remaining last bound, it is enough to show that

$$
\widetilde{p}_{j}^{(\kappa)} \leq 2 p_{j}^{(\kappa)} \quad(j=1,2, \ldots)
$$

hold for large enough $\kappa$ because of $\sum_{j=1}^{\infty} p_{j}^{(\kappa) 2}=O\left(\kappa^{d}\right)$. In fact, in the expression

$$
\widetilde{p}_{j}^{(\kappa)}=\frac{r_{\kappa} \widetilde{g}_{j}^{(\kappa)}}{1-r_{\kappa} \widetilde{g}_{j}^{(\kappa)}} \frac{1+\left(\widetilde{r}_{\kappa}-r_{\kappa}\right) / r_{\kappa}}{1-\left(\widetilde{r}_{\kappa}-r_{\kappa}\right) \widetilde{g}_{j}^{(\kappa)} /\left(1-r_{\kappa} \widetilde{g}_{j}^{(\kappa)}\right)},
$$

$\left(\widetilde{r}_{\kappa}-r_{\kappa}\right) / r_{\kappa}=O\left(\kappa^{-d / 2}\right)$ and $\left|\left(\widetilde{r}_{\kappa}-r_{\kappa}\right) \widetilde{g}_{j}^{(\kappa)} /\left(1-r_{\kappa} \widetilde{g}_{j}^{(\kappa)}\right)\right| \leq\left(\widetilde{r}_{\kappa}-r_{\kappa}\right) /\left(1-r_{\kappa} g_{1}^{(\kappa)}\right)=O\left(\kappa^{1-d / 2}\right)$ hold. Because of $\widetilde{g}_{j}^{(\kappa)} \leq g_{j}^{(\kappa)}$, we also have $r_{\kappa} \widetilde{g}_{j}^{(\kappa)} /\left(1-r_{\kappa} \widetilde{g}_{j}^{(\kappa)}\right) \leq p_{j}^{(\kappa)}$. Thus we get (3.6). 
It is obvious now that the next Lemma can be derived along the same line of reasoning as the proof of Lemma 3.4.

Lemma 3.6. For large $\kappa$, one gets the asymptotics

$$
\widetilde{\Xi}_{\kappa}(\beta, \mu)=\sqrt{\frac{2 \pi \beta \mu}{e^{2} \kappa^{d}}} \frac{\beta^{d-1} e^{\kappa^{d}\left(\beta \mu-\log \tilde{r}_{\kappa}\right)^{2} / 2 \beta \lambda}}{\left(\beta^{d} \mu-\zeta(d) \lambda\right) \operatorname{Det}\left(1-\widetilde{r}_{\kappa} \widetilde{G}_{\kappa}(\beta)\right)}(1+o(1)) .
$$

In order to calculate the limit of $\widetilde{\Xi}_{\kappa}(\beta, \mu) / \Xi_{\kappa}(\beta, \mu)$, we use the following lemma, where we put

$$
\hat{g}_{0}^{(\kappa)}:=\left(\Omega_{\kappa}, \widetilde{G}_{\kappa}(\beta) \Omega_{\kappa}\right)+\widetilde{r}_{\kappa}\left(\Omega_{\kappa}, \widetilde{G}_{\kappa}(\beta) Q_{\kappa}\left(1-\widetilde{r}_{\kappa} Q_{\kappa} \widetilde{G}_{\kappa}(\beta) Q_{\kappa}\right)^{-1} Q_{\kappa} \widetilde{G}_{\kappa}(\beta) \Omega_{\kappa}\right) .
$$

Lemma 3.7: For large $\kappa$, one gets

(i)

$$
\widetilde{r}_{\kappa}-r_{\kappa}=\left(1-\widetilde{g}_{0}^{(\kappa)}\right)(1+o(1))=\hat{O}\left(\kappa^{-d / 2}\right)
$$

and

(ii)

$$
1-\widetilde{r}_{\kappa} \hat{g}_{0}^{(\kappa)}=\left(1-\widetilde{r}_{\kappa} \widetilde{g}_{0}^{(\kappa)}\right)(1+o(1)) .
$$

Proof: From Lemma 3.5 and Proposition 2.4(b), we have $\widetilde{r}_{\kappa} \widetilde{g}_{0}^{(\kappa)}-r_{\kappa}=o\left(\kappa^{-d}\right)$. Hence (i) follows from $\widetilde{r}_{\kappa}-r_{\kappa}=\widetilde{r}_{\kappa}\left(1-\widetilde{g}_{0}^{(\kappa)}\right)+\widetilde{r}_{\kappa} \widetilde{g}_{0}^{(\kappa)}-r_{\kappa}, \widetilde{r}_{\kappa}=1+O\left(\kappa^{d / 2}\right)$ and Lemma 2.3.

By virtue of Lemma 2.3, we get

$$
\begin{aligned}
\widetilde{g}_{0}^{(\kappa)}-\hat{g}_{0}^{(\kappa)} & =\left(W_{\kappa}^{*} \Omega_{\kappa}, W_{\kappa}^{*} Q_{\kappa}\left[\left(\widetilde{g}_{0}^{(\kappa)}-Q_{\kappa} \widetilde{G}_{\kappa}(\beta) Q_{\kappa}\right)^{-1}-\left(\widetilde{r}_{\kappa}^{-1}-Q_{\kappa} \widetilde{G}_{\kappa}(\beta) Q_{\kappa}\right)^{-1}\right] Q_{\kappa} W_{\kappa} W_{\kappa}^{*} \Omega_{\kappa}\right) \\
& =\left(W_{\kappa}^{*} \Omega_{\kappa}, W_{\kappa}^{*} Q_{\kappa}\left(\widetilde{g}_{0}^{(\kappa)}-Q_{\kappa} \widetilde{G}_{\kappa}(\beta) Q_{\kappa}\right)^{-1}\left(\widetilde{r}_{\kappa}^{-1}-\widetilde{g}_{0}^{(\kappa)}\right)\left(\widetilde{r}_{\kappa}^{-1}-Q_{\kappa} \widetilde{G}_{\kappa}(\beta) Q_{\kappa}\right)^{-1} Q_{\kappa} W_{\kappa} W_{\kappa}^{*} \Omega_{\kappa}\right),
\end{aligned}
$$

which yields $\widetilde{g}_{0}^{(\kappa)}-\hat{g}_{0}^{(\kappa)} \geq 0$ since $\widetilde{r}_{\kappa}<\widetilde{g}_{0}^{(\kappa)-1}$. Recall that $\|\widetilde{X}\| \leq 1$ and $\left\|W_{\kappa}^{*} \Omega_{0}^{(\kappa)}\right\|=O\left(\kappa^{-d / 4}\right)$. [See (2.19) and its next line.] Then we also get from (3.8) that

$$
\begin{aligned}
\widetilde{g}_{0}^{(\kappa)}-\hat{g}_{0}^{(\kappa)} & \leq \frac{1-\widetilde{r}_{\kappa} \widetilde{g}_{0}^{(\kappa)}}{1-\widetilde{r}_{\kappa} g_{1}^{(\kappa)}}\left(W_{\kappa}^{*} \Omega_{0}^{(\kappa)}, W_{\kappa}^{*} Q_{\kappa}\left(\widetilde{g}_{0}^{(\kappa)}-Q_{\kappa} \widetilde{G}_{\kappa}(\beta) Q_{\kappa}\right)^{-1} Q_{\kappa} W_{\kappa} W_{\kappa}^{*} \Omega_{0}^{(\kappa)}\right) \\
& \leq \frac{1-\widetilde{r}_{\kappa} \widetilde{g}_{0}^{(\kappa)}}{1-\widetilde{r}_{\kappa} g_{1}^{(\kappa)}}\|\widetilde{X}\|\left\|W_{\kappa}^{*} \Omega_{0}^{(\kappa)}\right\|^{2}=\left(1-\widetilde{r}_{\kappa} \widetilde{g}_{0}^{(\kappa)}\right) O\left(\kappa^{1-d / 2}\right) .
\end{aligned}
$$

Hence we obtain the asymptotics (ii),

$$
1-\widetilde{r}_{\kappa} \hat{g}_{0}^{(\kappa)}=1-\widetilde{r}_{\kappa} \widetilde{g}_{0}^{(\kappa)}+\widetilde{r}_{\kappa}\left(\widetilde{g}_{0}^{(\kappa)}-\hat{g}_{0}^{(\kappa)}\right)=\left(1-\widetilde{r}_{\kappa} \widetilde{g}_{0}^{(\kappa)}\right)(1+o(1)) .
$$

Now, taking into account (3.4) and (3.7), we can find the asymptotics of the generating functional (1.25), 


$$
\begin{aligned}
E_{\kappa, \beta, \mu}\left[e^{-\langle f, \xi)}\right]= & \frac{\widetilde{\Xi}_{\kappa}(\beta, \mu)}{\Xi_{\kappa}(\beta, \mu)}=\exp \left(\frac{\kappa^{d}}{2 \beta \lambda}\left(2 \beta \mu-\log r_{\kappa} \widetilde{r}_{\kappa}\right) \log \frac{r_{\kappa}}{\widetilde{r}_{\kappa}}\right) \frac{\operatorname{det}\left[1-\widetilde{r}_{\kappa} Q_{\kappa} \widetilde{G}_{\kappa}(\beta) Q_{\kappa}\right]}{\operatorname{det}\left[1-\widetilde{r}_{\kappa} \widetilde{G}_{\kappa}(\beta)\right]} \\
& \times \frac{\operatorname{det}\left[1-\widetilde{r}_{\kappa} Q_{\kappa} G_{\kappa}(\beta) Q_{\kappa}\right]}{\operatorname{det}\left[1-\widetilde{r}_{\kappa} Q_{\kappa} \widetilde{G}_{\kappa}(\beta) Q_{\kappa}\right]} \frac{\operatorname{det}\left[1-r_{\kappa} Q_{\kappa} G_{\kappa}(\beta) Q_{\kappa}\right]}{\operatorname{det}\left[1-\widetilde{r}_{\kappa} Q_{\kappa} G_{\kappa}(\beta) Q_{\kappa}\right] \operatorname{det}\left[1-r_{\kappa} Q_{\kappa} G_{\kappa}(\beta) Q_{\kappa}\right]}(1+o(1)) .
\end{aligned}
$$

By virtue of Lemma 3.7(i), for the exponent of the first factor, we have

$$
\frac{\kappa^{d}}{2 \beta \lambda}\left(2 \beta \mu-\log r_{\kappa} \widetilde{\kappa}_{\kappa}\right) \log \frac{r_{\kappa}}{\widetilde{r}_{\kappa}}=-\frac{\mu \kappa^{d}}{\lambda}\left(1-\widetilde{g}_{0}^{(\kappa)}\right)(1+o(1)) .
$$

For the second factor, we use the Feshbach formula, which claims

$$
\operatorname{det} A=\operatorname{det} B \operatorname{det}\left(C-K^{T} B^{-1} K\right),
$$

where

$$
A=\left(\begin{array}{cc}
B & -K \\
-K^{T} & C
\end{array}\right)=\left(\begin{array}{cc}
1 & 0 \\
-K^{T} B^{-1} & 1
\end{array}\right)\left(\begin{array}{cc}
B & 0 \\
0 & C-K^{T} B^{-1} K
\end{array}\right)\left(\begin{array}{cc}
1 & -B^{-1} K \\
0 & 1
\end{array}\right) .
$$

This formula and Lemma 3.7(ii) yield

$$
\begin{aligned}
& \frac{\operatorname{det}\left[1-\widetilde{r}_{\kappa} Q_{\kappa} \widetilde{G}_{\kappa}(\beta) Q_{\kappa}\right]}{\operatorname{det}\left[1-\widetilde{r}_{\kappa} \widetilde{G}_{\kappa}(\beta)\right]} \\
& =\frac{1}{1-\widetilde{r}_{\kappa}\left(\Omega_{0}^{(\kappa)}, \widetilde{G}_{\kappa}(\beta) \Omega_{0}^{(\kappa)}\right)-\left(\Omega_{0}^{(\kappa)}, \widetilde{r}_{\kappa} \widetilde{G}_{\kappa}(\beta) Q_{\kappa}\left(1-\widetilde{r}_{\kappa} Q_{\kappa} \widetilde{G}_{\kappa}(\beta) Q_{\kappa}\right)^{-1} Q_{\kappa} \widetilde{r}_{\kappa} \widetilde{G}_{\kappa}(\beta) \Omega_{0}^{(\kappa)}\right)} \\
& =1 /\left(1-\widetilde{r}_{\kappa} \hat{g}_{0}^{(\kappa)}\right)=(1+o(1)) /\left(1-\widetilde{r}_{\kappa} \widetilde{g}_{0}^{(\kappa)}\right) .
\end{aligned}
$$

Since

$$
\frac{\operatorname{det}\left[1-r_{\kappa} G_{\kappa}(\beta)\right]}{\operatorname{det}\left[1-r_{\kappa} Q_{\kappa} G_{\kappa}(\beta) Q_{\kappa}\right]}=1-r_{\kappa},
$$

then Proposition 2.4(b) and Lemma 3.5 yields for the product of factors in (3.9),

$$
\text { (the second factor }) \times(\text { the last factor }) \rightarrow 1
$$

in the limit $\kappa \rightarrow \infty$.

Now, since Lemma 3.5 and (2.11) give

$$
\begin{aligned}
& \left\|\sqrt{1-e^{-f}} \frac{\widetilde{r}_{\kappa} Q_{\kappa} G_{\kappa}(\beta) Q_{\kappa}}{1-\widetilde{r}_{\kappa} Q_{\kappa} G_{\kappa}(\beta) Q_{\kappa}} \sqrt{1-e^{-f}}-K_{f}\right\|_{1} \\
& \quad \leq\left\|\sqrt{1-e^{-f}} \frac{\widetilde{r}_{\kappa} Q_{\kappa} G_{\kappa}(\beta) Q_{\kappa}}{1-\widetilde{r}_{\kappa} Q_{\kappa} G_{\kappa}(\beta) Q_{\kappa}} \sqrt{1-e^{-f}}-\sqrt{1-e^{-f}} \frac{Q_{\kappa} G_{\kappa}(\beta) Q_{\kappa}}{1-Q_{\kappa} G_{\kappa}(\beta) Q_{\kappa}} \sqrt{1-e^{-f}}\right\|_{1} \\
& \quad+\left\|\sqrt{1-e^{-f}} \frac{Q_{\kappa} G_{\kappa}(\beta) Q_{\kappa}}{1-Q_{\kappa} G_{\kappa}(\beta) Q_{\kappa}} \sqrt{1-e^{-f}}-K_{f}\right\|_{1} \\
& \quad \leq\left|\widetilde{r}_{\kappa}-1\right|\left\|\sqrt{1-e^{-f}} \frac{Q_{\kappa} G_{\kappa}(\beta) Q_{\kappa}}{1-Q_{\kappa} G_{\kappa}(\beta) Q_{\kappa}} \sqrt{1-e^{-f}}\right\|_{1}\left\|\left(1-\widetilde{r}_{\kappa} Q_{\kappa} G_{\kappa}(\beta) Q_{\kappa}\right)^{-1}\right\|
\end{aligned}
$$




$$
+\left\|\sqrt{1-e^{-f}} \frac{Q_{\kappa} G_{\kappa}(\beta) Q_{\kappa}}{1-Q_{\kappa} G_{\kappa}(\beta) Q_{\kappa}} \sqrt{1-e^{-f}}-K_{f}\right\|_{1} \rightarrow 0
$$

for $\kappa \rightarrow \infty$, we obtain the limit

$$
\begin{aligned}
\frac{\operatorname{det}\left[1-\widetilde{r}_{\kappa} Q_{\kappa} G_{\kappa}(\beta) Q_{\kappa}\right]}{\operatorname{det}\left[1-\widetilde{r}_{\kappa} Q_{\kappa} \widetilde{G}_{\kappa}(\beta) Q_{\kappa}\right]} & =\frac{1}{\operatorname{det}\left[1+\widetilde{r}_{\kappa} Q_{\kappa}\left(G_{\kappa}(\beta)-\widetilde{G}_{\kappa}(\beta)\right) Q_{\kappa}\left(1-\widetilde{r}_{\kappa} Q_{\kappa} G_{\kappa}(\beta) Q_{\kappa}\right)^{-1}\right]} \\
& =\operatorname{det}\left[1+\widetilde{r}_{\kappa} \sqrt{1-e^{-f}} Q_{\kappa} G_{\kappa}(\beta) Q_{\kappa}\left(1-\widetilde{r}_{\kappa} Q_{\kappa} G_{\kappa}(\beta) Q_{\kappa}\right)^{-1} \sqrt{1-e^{-f}}\right]^{-1} \\
& \rightarrow \operatorname{det}\left[1+K_{f}\right]^{-1}
\end{aligned}
$$

for the third factor in (3.9). Here we have used the cyclicity of the Fredholm determinant.

Lemma 3.7(i), (3.3), (2.3), and Proposition 2.4(b) yield

$$
\begin{aligned}
\frac{\operatorname{det}\left[1-r_{\kappa} Q_{\kappa} G_{\kappa}(\beta) Q_{\kappa}\right]}{\operatorname{det}\left[1-\widetilde{r}_{\kappa} Q_{\kappa} G_{\kappa}(\beta) Q_{\kappa}\right]} & =\frac{1}{\operatorname{det}\left[1-\left(\widetilde{r}_{\kappa}-r_{\kappa}\right) Q_{\kappa} G_{\kappa}(\beta) Q_{\kappa}\left(1-r_{\kappa} Q_{\kappa} G_{\kappa}(\beta) Q_{\kappa}\right)^{-1}\right]} \\
& =\exp \left(\frac{\widetilde{r}_{\kappa}-r_{\kappa}}{r_{\kappa}} \operatorname{Tr} \frac{r_{\kappa} Q_{\kappa} G_{\kappa}(\beta) Q_{\kappa}}{1-r_{\kappa} Q_{\kappa} G_{\kappa}(\beta) Q_{\kappa}}+O(1)\right) \\
& =\exp \left(\left(1-\widetilde{g}_{0}^{(\kappa)}\right)\left[\kappa^{d} \frac{\beta \mu-\log r_{\kappa}}{\beta \lambda}-\frac{r_{\kappa}}{1-r_{\kappa}}\right]+O(1)\right) \\
& =\exp \left\{\left(1-\widetilde{g}_{0}^{(\kappa)}\right) \kappa^{d} \zeta(d)(1+o(1)) / \beta^{d}\right\} .
\end{aligned}
$$

Thus, by Lemma 2.3 and (2.11) we get for the product in (3.9),

(the first factor) $\times($ the fourth factor $)$

$$
=\exp \left(-\frac{\kappa^{d / 2}(1+o(1))}{\pi^{d / 2}} \frac{\beta^{d} \mu-\zeta(d) \lambda}{\beta^{d} \lambda}\left(\sqrt{1-e^{-f}},\left(1+K_{f}\right)^{-1} \sqrt{1-e^{-f}}\right)\right) .
$$

Now Theorem 1.2(ii) follows by collecting in (3.9) the asymptotics of factors that we find above.

\section{PROOF OF THEOREM 1.8}

We start with the grand-canonical expectation value of the total number of MF interacting bosons in the WHT (1.31),

$$
N_{\kappa, \lambda}(\beta, \mu):=\kappa^{d} \rho_{\kappa, \lambda}^{(\mathrm{tot})}(\beta, \mu)=\frac{1}{\beta \Xi_{\kappa}(\beta, \mu)} \frac{\partial}{\partial \mu} \Xi_{\kappa}(\beta, \mu),
$$

where we use for $\Xi_{\kappa}(\beta, \mu)$ expression (2.2) after the $z$-integration where the values of $s$ and $r$ are not fixed yet. The differentiation with respect to $\mu$ can be converted into differentiation with respect to $x$ in the Fredholm determinant. Then integrating by parts we obtain

$$
N_{\kappa, \lambda}(\beta, \mu)=s-\frac{i \kappa^{d}}{\beta \lambda} R(r, s)
$$

where 


$$
\begin{aligned}
R(r, s):= & \int_{\mathbb{R}} d x \frac{x e^{-i s x-\kappa^{d} x^{2} / 2 \beta \lambda}}{\operatorname{det}\left[1-\left(e^{i x}-1\right) r G_{\kappa}(\beta)\left(1-r G_{\kappa}(\beta)\right)^{-1}\right]} \\
& \times\left[\int_{\mathbb{R}} d x \frac{e^{-i s x-\kappa^{d} x^{2} / 2 \beta \lambda}}{\operatorname{det}\left[1-\left(e^{i x}-1\right) r G_{\kappa}(\beta)\left(1-r G_{\kappa}(\beta)\right)^{-1}\right]}\right]^{-1} .
\end{aligned}
$$

Then we put $s=s_{\kappa}$ and $r=r_{\kappa}$ such that (2.3) holds, and if we prove $R\left(r_{\kappa}, s_{\kappa}\right)=o(1)$, then (1.32) follows as a consequence of this asymptotics.

To this end notice that for the case (i) $\mu<\mu_{c}(\beta)$, we get $R\left(r_{\kappa}, s_{\kappa}\right)=O\left(\kappa^{-d / 2}\right)$ from the estimates in the proof of Lemma 3.1.

For the case (ii) $\mu>\mu_{c}(\beta)$, using the notations of the proof of Lemma 3.4, one obtains for the first factor in (4.1),

$$
\int_{\mathrm{R}} d x \frac{x e^{-i s_{\kappa} x-\kappa^{d} x^{2} / 2 \beta \lambda}}{\operatorname{det}\left[1-\left(e^{i x}-1\right) r_{\kappa} G_{\kappa}(\beta)\left(1-r_{\kappa} G_{\kappa}(\beta)\right)^{-1}\right]}=-i \varepsilon_{0} \mathcal{I}_{1}+o\left(\mathcal{I}_{1}\right) .
$$

Therefore, we get that $R\left(r_{\kappa}, s_{\kappa}\right)=O\left(\kappa^{-d}\right)$.

The other properties stated in the Theorem 1.8 follow straightforwardly from Sec. II D and the line of reasoning developed for the proof of Proposition 2.4.

\section{CONCLUDING REMARKS AND CONJECTURES}

In the present paper we consider a model of mean-field interacting boson gas in traps described by the harmonic potential. For this model we study the position distribution of the constituent bosons in the WHT limit by means of the RPF method.

It is shown that there are two phases distinguished by the boson condensation. In one domain of parameters, the resulting generating functional for the RPF is the same as for the noninteracting boson gas, for unconventional values of the IBG parameters. Whereas in another domain, the generating functional describes divergence of the density due to the localization of macroscopic number of particles.

Our results are obtained via analysis of the generating functional. We do not intend to start the analysis using the characteristic functional here. However, we would like to mention a topic on the central limit theorem as a conjecture.

Let us consider $E_{\kappa, \beta, \mu}\left[e^{-\langle f, \xi)}\right]$ for small $f \in C_{0}\left(\mathbb{R}^{d}\right)$ in the sense of the sup-norm $\|\cdot\|_{\infty}$. By Theorem 1.2(ii) we obtain for $\mu>\mu_{\lambda, c}(\beta)$ that

$$
E_{\kappa, \beta, \mu}\left[e^{-\langle f, \xi)}\right]=\exp \left\{-\frac{\kappa^{d / 2}}{\pi^{d / 2}} \frac{\mu-\mu_{\lambda, c}(\beta)}{\lambda}\left[\int_{\mathrm{R}^{d}} f(x) d x-\frac{1}{2}\left\langle f,(1+G(\beta))(1-G(\beta))^{-1} f\right\rangle+\cdots\right]\right\} .
$$

This leads us to infer that the equality would also holds for $i \epsilon f$ instead of $-f$, where $\epsilon$ $:=(\pi / \kappa) \sqrt[d / 4]{\lambda /\left(\mu-\mu_{\lambda, c}(\beta)\right)}$. In particular, we expect that the following central limit theorem should be true:

Conjecture 5.1: Let $\xi_{\kappa}$ be the random point measure on $\mathbb{R}^{d}$ with distribution given by $\nu_{\kappa, \beta, \mu}$ for $\kappa>0$, and $\mathfrak{L}$ be the Lebesgue measure on $\mathbb{R}^{d}$. Then for $\kappa \rightarrow \infty$ the random field

$$
\frac{\pi^{d / 4}}{\kappa^{d / 4}}\left(\frac{\lambda}{\mu-\mu_{\lambda, c}(\beta)}\right)^{1 / 2}\left(\xi_{\kappa}-\frac{\kappa^{d / 2}}{\pi^{d / 2}} \frac{\mu-\mu_{\lambda, c}(\beta)}{\lambda} \mathfrak{L}\right)
$$

converges in distribution to the Gaussian random field on $\mathbb{R}^{d}$ with covariance

$$
(1+G(\beta))(1-G(\beta))^{-1} .
$$

We finish by some remarks about the method of the RPF approach to the BEC in the WHT limit used in the present paper. 
(i) It could be applied to a general "nonquadratic" mean-field interaction $U_{\Phi}:=\kappa^{d} \Phi\left(n / \kappa^{d}\right)$, where $\Phi: x \in \mathbb{R} \mapsto \mathbb{R}$ is a piecewise differentiable continuous function bounded from below, as well as to the van der Waals particle interaction, which is more local than the mean field. ${ }^{2}$ We guess that for this kind of interaction the particle distribution will spread as $\kappa^{\alpha}$ with some large $\alpha$ even for condensed particles.

(ii) The method has to be compared to the scaled external field perturbation of BEC considered in Refs. 2 and 15. We suppose that this could clarify the concept of the choice of "effective" volume, since it is important for description of the local particle density measured in the WHT limit BEC experiments as well as its definition of the mean-field interaction. ${ }^{3,10,14}$

\section{ACKNOWLEDGMENTS}

H.T. thanks MEXT for the financial support under the Grant-in-Aid for Exploratory Research under Grant No. 17654021. V.A.Z. is thankful to Mathematical Department of the Kanazawa University for a warm hospitality and financial support.

We are thankful the referee for useful remarks.

\section{APPENDIX: MISCELLANEOUS FORMULAS}

$$
\begin{array}{r}
\frac{x}{1-e^{-x}} \leq 1+x \leq 2 \vee(2 x) \text { for } x>0 . \\
0 \leq\left(\frac{2 x}{1-e^{-2 x}}\right)^{d / 2}-1 \leq A x \text { for } x \in[0,1], \\
0 \leq\left(\frac{1}{1-e^{-2 x}}\right)^{d / 2}-1 \leq B e^{-2 x} \text { for } x \geq 1,
\end{array}
$$

where $A$ and $B$ are constants depending only on $d$.

$$
\begin{aligned}
& 1-e^{-x} \leq x \text { for } x \geq 0 \text {. } \\
& e^{-x}-e^{-y} \leq \frac{1}{e}\left(\frac{y}{x}-1\right) \text { for } 0<x \leq y . \\
& \tanh x \leq x \wedge 1 \text { for } x \geq 0 \text {. } \\
& |\operatorname{coth} x-1| \leq \frac{2 e}{e-1} e^{-x} \quad \text { for } x \geq 1 \text {. } \\
& 1 \leq \frac{\sinh x}{x} \leq 1+x^{2} \text { for } x \in[0,1] \\
& \frac{1}{\sinh x} \leq \frac{2}{1-1 / e^{2}} e^{-x} \quad \text { for } x \geq 1 \text {. } \\
& |-\log (1-x)-x| \leq \frac{x^{2}}{2(1-x)} \quad \text { for } x<1
\end{aligned}
$$


${ }^{1}$ Daley, D. J. and Vere-Jones, D., An Introduction to the Theory of Point Processes (Springer, Berlin, 1988).

${ }^{2}$ de Smedt, Ph. and Zagrebnov, V. A., "van der Waals limit of an interacting Bose gas in a weak external field," Phys. Rev. A 35, 4763 (1987).

${ }^{3}$ Dalfovo, F., Giorgini, S., Pitaevskii, L. P., and Stringari, S., "Theory of Bose-Einstein condensation in trapped gases," Rev. Mod. Phys. 71, 463 (1999).

${ }^{4}$ Eisenbaum, N., "A Cox process involved in the Bose-Einstein condensation," Ann. Henri Poincare 9, 1123 (2008).

${ }^{5}$ Freudenberg, W., "Characterization of states of infinite boson systems. II. On the existence of the conditional reduced density matrix," Commun. Math. Phys. 137, 461 (1991).

${ }^{6}$ Fichtner, K.-H. and Freudenberg, W., "Characterization of states of infinite boson systems. I. On the construction of states of boson systems," Commun. Math. Phys. 137, 315 (1991).

${ }^{7}$ Kato, T., Perturbation Theory for Linear Operators (Springer-Verlag, Heidelberg, 1966).

${ }^{8}$ Lauwers, J., Verbeure, A., and Zagrebnov, V. A., "Bose-Einstein condensation for homogeneous interacting system with a one-particle spectral gap," J. Stat. Phys. 112, 397 (2003).

${ }^{9}$ Lewis, J. and Pulè, J. V., "The equilibrium states of the free boson gas," Commun. Math. Phys. 36, 1 (1974).

${ }^{10}$ Lieb, E. H., Seiringer, R., Solovej, J. P., and Yngvason, J., The Mathematics of the Bose Gas and its Condensation (Birkhäuser, Basel, 2005).

${ }^{11}$ Lytvynov, E., "Fermion and boson random point processes as particle distributions of infinite free Fermi and Bose gases of finite density," Rev. Math. Phys. 14, 1073 (2002).

${ }^{12}$ Macchi, O., "The coincidence approach to stochastic point processes," Adv. Appl. Probab. 7, 83 (1975).

${ }^{13}$ Macchi, O., "The fermion process-a model of stochastic point process with repulsive points," in Transactions of the Seventh Prague Conference on Information Theory, Statistical Decision Functions, Random Processes and of the Eighth European Meeting of Statisticians, Prague, Czech Republic, 1974 (Reidel, Dordrecht, 1977), Vol. A, pp. 391-398.

${ }^{14}$ Pethick, C. J. and Smith, H., Bose-Einstein condensation in dilute gases (Cambridge University Press, Cambridge, 2002).

${ }^{15}$ Pulè, J. V., "The free boson gas in a weak external potential," J. Math. Phys. 24, 138 (1983).

${ }^{16}$ Reed, M. and Simon, B., Methods of Modern Mathematical"Physics: Analysis of Operators (Academic, London, 1978), Vol. IV.

${ }^{17}$ Shirai, T. and Takahashi, Y., "Random point fields associated with certain Fredholm determinants. I. Fermion, Poisson and boson point processes," J. Funct. Anal. 205, 414 (2003).

${ }^{18}$ Tamura, H. and Ito, K. R., "A canonical ensemble approach to the fermion/boson random point processes and its applications," Commun. Math. Phys. 263, 353 (2006).

${ }^{19}$ Tamura, H. and Ito, K. R., "A random point field related to Bose-Einstein condensation,” J. Funct. Anal. 243, 207 (2007).

${ }^{20}$ Tamura, H. and Ito, K. R., "Random point fields for para-particles of any order," J. Math. Phys. 48, 023301 (2007).

${ }^{21}$ Vandevenne, L., Verbeure, A., and Zagrebnov, V. A., "Equilibrium states for the Bose gas," J. Math. Phys. 45, 1606 (2004).

${ }^{22}$ Vere-Jones, D., "A generalization of permanents and determinants," Linear Algebra and its Applications 111, 119 (1988).

${ }^{23}$ Zagrebnov, V. A., Topics in the Theory of Gibbs Semigroups, Leuven Notes in Mathematical and Theoretical Physics Vol. 10 (Leuven University Press, Leuven, 2002).

${ }^{24}$ Zagrebnov, V. A. and Bru, J.-B., "The Bogoliubov model of weakly imperfect Bose gas,” Phys. Rep. 350, 291 (2001). 